\title{
Optimal Online Sensing Sequence in Multichannel Cognitive Radio Networks
}

\author{
Hyoil Kim, Member, IEEE, and Kang G. Shin, Fellow, IEEE
}

\begin{abstract}
We address the problem of rapidly discovering spectrum opportunities for seamless service provisioning in cognitive radio networks (CRNs). In particular, we focus on multichannel communications via channel-bonding with heterogeneous channel characteristics of ON/OFF patterns, sensing time, and channel capacity. Using dynamic programming (DP), we derive an optimal online sensing sequence incurring a minimal opportunity-discovery delay, and propose a suboptimal sequence that presents a nearoptimal performance while incurring significantly less computational overhead than the DP algorithm. To facilitate fast opportunity discovery, we also propose a channel-management strategy that maintains a list of backup channels to be used at building the optimal sequence. A hybrid of maximum likelihood (ML) and Bayesian inference is introduced as well for flexible estimation of ON/OFF channel-usage patterns, which selectively chooses the better between the two according to the frequency of sensing and ON/OFF durations. The performance of the proposed schemes, in terms of the opportunity-discovery delay, is evaluated via in-depth simulation, and for the scenarios we considered, the proposed suboptimal sequence achieves a near-optimal performance with only an average of 0.5 percent difference from the optimal delay, and outperforms the previously proposed probabilistic scheme by up to 50.1 percent. In addition, the backup channel update scheme outperforms the no-update case by up to 49.9 percent.
\end{abstract}

Index Terms-Cognitive radio, spectrum sensing, sensing sequence, backup channels, candidate channels, Bayesian estimation

\section{INTRODUCTION}

$\mathrm{D}$ YNAMIC Spectrum Access (DSA) is a key concept to solve the problem of wireless spectrum scarcity, rooted from inefficient spectrum utilization by the current static resource allocation policy. DSA can enhance spectrum utilization by allowing (unlicensed) secondary users (SUs), equipped with cognitive radios (CRs), to utilize the spectrum whitespaces (also called spectrum opportunities) during which spectrum bands (or channels) are left unused by their (licensed) primary users (PUs).

Spectrum opportunities are discovered by spectrum sensing that monitors channels to determine the presence/absence of PUs in them via PU signal detection. If thus-discovered idle channels are utilized by SUs, they are referred to as in-band channels; channels other than in-band channels are called out-of-band channels. Hence, opportunity discovery is an act of sensing out-of-band channels (called out-of-band sensing [2]) to locate whitespaces in them.

In this paper, we consider a CR network (CRN) that collectively utilizes multiple idle channels to serve the total spectrum demand of SUs in the CRN ( $B_{r e q}$ in Section 4), which may be achieved by combining a few high-capacity idle channels or many low-capacity idle channels. In this scenario, the CRN discovers only the necessary amount of whitespaces to meet the given bandwidth requirement,

- H. Kim is with the School of Electrical and Computer Engineering, Ulsan National Institute of Science and Technology, UNIST-gil 50, Ulsan 689798, Korea.E-mail:hkim@unist.ac.kr.

- K.G. Shin is with the Department of Electrical Engineering and Computer Science, University of Michigan, Computer Science and Engineering Building, 2260 Hayward Street, Ann Arbor, MI 48109-2121.

E-mail:kgshin@eecs.umich.edu.

Manuscript received 25 Sept. 2010; revised 11 Aug. 2011; accepted 19 Apr. 2012; published online 1 May 2012.

For information on obtaining reprints of this article, please send e-mail to: tmc@computer.org, and reference IEEECS Log Number TMC-2010-09-0444. Digital Object Identifier no. 10.1109/TMC.2012.108. instead of maximizing the throughput of the SUs as in [3] [4]. Therefore, opportunity discovery is triggered only when the CRN experiences a shortage of whitespaces due to the return of PUs ${ }^{1}$ at one of the in-band channels which forces SUs to immediately vacate the channel (called channel vacation).

At channel vacation, fast opportunity discovery is essential to seamless service provisioning for the SUs, because the CRN may not provide its service in full strength with a shortage of bandwidth. Since the discovery of new whitespaces incurs a nontrivial delay, it is desired to derive an optimal sequence of sensing out-of-band channels that minimizes the latency in discovering additional idle channels to satisfy the total bandwidth requirement. Therefore, our objective is to minimize "interruption" of SUs' operation while maintaining their minimum bandwidth requirement.

\subsection{Contributions}

Our contribution in this paper is threefold. First, we propose an optimal sensing sequence that minimizes the latency of discovering available out-of-band channels to achieve the target amount of opportunities. We consider heterogeneous channel characteristics in terms of signal detection time, channel capacity, and the probability $\theta_{i}$ that channel $i$ is idle, and discuss the difference between offline and online sequences to show that the optimal sequence is an online sequence. Then, we derive the optimal online sequence using dynamic programming (DP), and show that the optimal sequence takes a simple form when channels have homogeneous capacities. To

1. The return of PUs is detected by sensing in-band channels (called inband sensing [5]), which is separate from and independent of out-of-band sensing. The most common approach for in-band sensing is to sample an inband channel periodically (e.g., sense once every 2 seconds with a sensing duration of 1-10 ms). 
overcome the computational complexity of the DP algorithm, we also propose a suboptimal sequence algorithm that shows a near-optimal performance while incurring insignificant overhead.

Next, we categorize out-of-band channels as backup or candidate channels, as introduced in IEEE 802.22 [6]. To promote faster discovery of idle channels, we sort and search backup channels at the time of opportunity discovery where the backup channels are specially chosen among those that are more probable to have whitespaces; out-of-band channels other than backup channels are called candidate channels, and they are not sensed until they are designated as backup channels. Using this concept, we propose an efficient mechanism that constructs a backup channel list (BCL) and dynamically updates its entries. The proposed scheme maintains a moderate size of the BCL and updates its entries by importing/exporting channels from/to the candidate channel list (CCL) with a small computational overhead.

Finally, we propose a strategy that estimates ON/OFF channel-usage patterns to predict channel availability, by selectively applying maximum likelihood (ML) and Bayesian estimation. We capture the tradeoff between two estimation techniques: The former is simple but its performance degrades greatly with infrequent samples; the latter requires more computation but performs better with a small number of samples [7]. In addition, our scheme considers imperfect sensing with nonzero probabilities of miss detection (PMD) and false alarms (PFA) in predicting $\theta_{i}$.

\subsection{Organization}

We first overview related work in Section 2, and introduce our system models and assumptions in Section 3. Section 4 describes the opportunity discovery mechanism via sequential sensing. In Section 5, we derive the optimal sensing sequence and a suboptimal sensing sequence that achieves a near-optimal performance with small computational overhead. Section 6 presents construction of the initial BCL and an BCL-update algorithm to keep the list up-to-date. Section 7 introduces a strategy to estimate ON/OFF channel-usage patterns using ML and Bayesian inference. The performance of the proposed schemes are evaluated in Section 8, and then the paper concludes in Section 9.

\section{Related Work}

Among a number of studies on spectrum sensing, several notable bodies of work are found to be related to fast opportunity discovery. Chang and Liu [8] proposed a strategy that optimally determines which channel to probe and when to transmit. Jiang et al. [9] investigated the optimal sensing sequence in a multichannel cognitive MAC protocol. Shu and Krunz [3] studied the problem of sequential sensing for throughput efficiency along with finding the optimal sensing time. Ahmad et al. [10] derived an optimal myopic policy in finding the best channel to sense per time slot. Fan and Jiang [11] investigated the impact of adaptive modulation on channel sensing order. Cheng and Zhuang [12] proposed a simple channel sensing order that senses channels in descending order of their achievable rates. However, all of the above considered the case where data transmission utilizes up to one channel at a time, while our paper considers the case where CR-to-CR transmission occurs on a multichannel environment via channel bonding. Although Lai et al. [4] considered a scenario in which SUs can sense and utilize more than one channel simultaneously, their focus was to maximize the throughput of the SUs, whereas ours is to minimize the duration of service interruption in case the minimum bandwidth requirement is specified.

Regarding the Bayesian estimation, Motamedi and Bahai [13] introduced Bayesian learning to predict the availability of a channel, where the learning process is simplified by assuming a geometric distribution for channel-usage patterns. In this paper, we use a general alternating renewal process and develop a multistage iterative Bayesian inference.

This paper builds upon our preliminary work presented at IEEE DySPAN 2008 [1], which is significantly extended by:

1. Capturing the fundamental difference between offline and online sensing sequences,

2. deriving the optimal sequence for heterogeneous channel characteristics via application of DP to the online sensing sequence,

3. proposing a near-optimal sequence incurring very little computational overhead, and

4. enhancing efficiency of the BCL update by sorting channels with a new metric called effective capacity.

\section{SYSTEM MOdEL}

\subsection{Network Model}

We focus on a single-hop CRN with a central controller (e.g., an access point) and a group of SUs, where the CRN utilizes a set of $M$ licensed channels from which it harvests the necessary amount of spectrum opportunities. The $M$ licensed channels are assumed to be determined and given a priori through an inter-CRN coexistence mechanism such as IEEE SCC 41 [14] and the coordinated channel allocation schemes [15], [16]. The inter-CRN coexistence scheme coordinates resource allocation between neighboring CRNs, which is necessary to avoid collision by simultaneous channel access, through which the licensed channels can be assigned to the CRNs in a nonoverlapping fashion. Since such schemes are beyond the scope of this paper, we focus on selecting backup channels from the given $M$ channels and optimally sequencing them at opportunity discovery.

Each SU is assumed to have been equipped with a single antenna, widely tunable to any combination of $M$ channels. That is, a SU can utilize possibly noncontiguous multiple idle channels at the same time, which is made possible by using signal processing techniques such as NC-OFDM [17]. Having one antenna per SU may help reduce the size of a secondary device and avoid potential interference between colocated antennas due to their close proximity [18]. A SU is assumed to act as either a spectrum sensor or a secondary transceiver, by dynamically reconfiguring itself.

We assume the central controller coordinates inter-SU transmission, spectrum sensing, and channel switching. The SU coordination is performed by a CR MAC protocol such as C-MAC [19] and OS-MAC [20], which is beyond the scope of this paper. Using the proposed sensing sequence in 

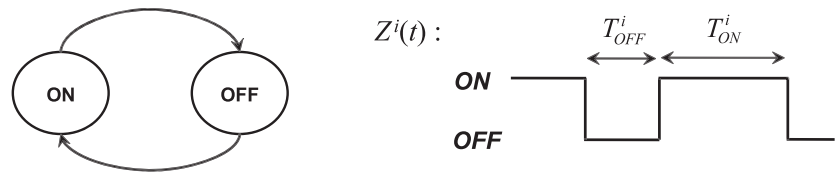

Fig. 1. Channel model: Alternating renewal process with ON and OFF states.

Section 5, the controller can assign as many SUs/sensors as necessary to achieve the PU protection requirements (e.g., PMD and PFA). Note that the optimality of the proposed sensing sequences is intact regardless of which MAC protocol is employed.

\subsection{Channel Model}

A channel is modeled as a renewal process alternating between $\mathrm{ON}$ and OFF states. The ON (OFF) state represents a time period within which a PU signal is present (absent). Once sensing finds a channel in its OFF state (i.e., an idle channel), SUs can utilize the channel until its next state transition to $\mathrm{ON}$ state. This type of channel model was introduced in [2], [13], [21], where its potential for modeling spectrum opportunities was demonstrated.

Fig. 1 illustrates the channel model. Suppose $i$ is the channel index $(i=1,2, \ldots, M)$, and let $Z^{i}(t)$ denote the state (ON or OFF) of channel $i$ at time $t$, such that

$$
\begin{cases}Z^{i}(t)=1, & \text { if channel } i \text { is ON (or busy) at } t, \\ Z^{i}(t)=0, & \text { otherwise. }\end{cases}
$$

For an alternating renewal channel [22], the sojourn times of $\mathrm{ON}$ and OFF states are represented by random variables $T_{O N}^{i}$ and $T_{O F F}^{i}$ with probability density functions (pdfs) $f_{T_{O N}^{i}}(t)$ and $f_{T_{O F F}^{i}}(t), t>0$, respectively. ON and OFF states are independent of each other.

Note that $f_{T_{O N}^{i}}(t)$ and $f_{T_{O F F}^{i}}(t)$ can be any distribution functions. For example, for exponentially distributed $\mathrm{ON} /$ OFF periods, we have

$$
f_{T_{O F F}^{i}}(t)=\psi_{O F F}^{i} e^{-\psi_{O F F}^{i} t}, f_{T_{O N}^{i}}(t)=\psi_{O N}^{i} e^{-\psi_{O N}^{i} t},
$$

where $E\left[T_{O N}^{i}\right]=1 / \psi_{O N}^{i}$ and $E\left[T_{O F F}^{i}\right]=1 / \psi_{O F F}^{i}$.

On the other hand, channel utilization, $u^{i} \in[0,1]$, defined as the average fraction of time during which channel $i$ is in $\mathrm{ON}$ state, is given as

$$
u^{i}=E\left[T_{O N}^{i}\right] /\left\{E\left[T_{O N}^{i}\right]+E\left[T_{O F F}^{i}\right]\right\} .
$$

$C_{i}$ is the capacity of channel $i$ which is a physical bandwidth or Shannon capacity, taken as a long-term average considering time-varying channel conditions (e.g., fading). Although instantaneous capacity can also be used in view of fast fading statistics at each instant, we avoid using such fast-changing channel conditions in achieving the target amount of opportunities because we do not want overly sensitive channel switching due to the short-term degradation of channel quality.

\subsection{Sensing Model}

When a SU acts as a spectrum sensor, it monitors channel $i$ during a certain time period, called sensing-time $T_{I}^{i}$, and determines the channel state between $\mathrm{ON}$ and OFF. Therefore, sensing is akin to a sampling process producing

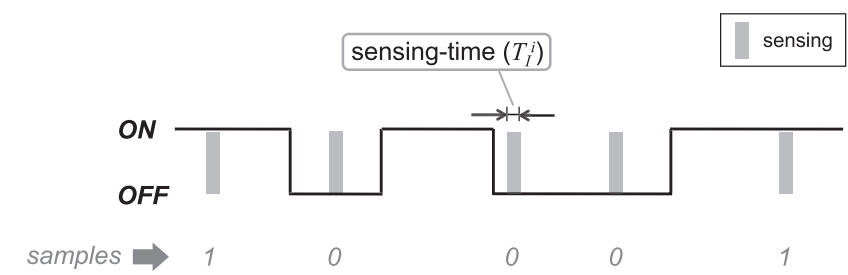

Fig. 2. Illustration of the sensing process on a channel.

a binary random sequence of $0(\mathrm{OFF})$ and $1(\mathrm{ON})$, as illustrated in Fig. 2. Note that sensing is not necessarily periodic. $T_{I}^{i}$ is assumed small relative to $E\left[T_{O F F}^{i}\right]$ and $E\left[T_{O N}^{i}\right]$ such that channel $i^{\prime}$ s state remains unchanged during the sensing-time.

The value of $T_{I}^{i}$ is determined by the underlying detection method (e.g., energy or feature detection) and the type of PU signals [23], and thus it varies with channels. In this paper, we assume $T_{I}^{i}$ is determined a priori and fixed, due to the following reasons. First, in many CR scenarios we take a conservative approach in choosing the detection method (e.g., energy and feature detection) and the sensing time (i.e., $T_{I}^{i}$ ) to achieve the PU protection requirements (e.g., PMD and PFA). This is because CRNs generally do not have any feedback from the PU's network, and thus we should protect them (both PU transmitter and receiver) based on the worst case scenario assuming PU receivers are located at the edge of their coverage. The IEEE 802.22's approach in determining the sensing sensitivity of $-114 \mathrm{dBm}$ for DTV protection is a good example of such approach. Then, as shown in [5], we can derive a fixed value of $T_{I}^{i}$ that minimizes the sensing overhead while achieving all the PU protection requirements, which becomes the value of $T_{I}^{i}$ in this paper.

We assume each out-of-band sensing requires all SUs to sense a channel simultaneously (called collaborative sensing [24], [25]) to exploit location diversity of sensors for better detection performance. This assumption is reasonable for a mid-sized CRN with up to 20 SUs, because the desirable number of collaborative sensors is known to be 10-20 [26]. For a large-scale CRN with more than 20 SUs, however, a part of SUs may participate in out-of-band sensing while others continue to utilize in-band channels. In this case, outof-band sensing should be performed by all the SUs vacated from an in-band channel plus some extra SUs in other inband channels to recruit a sufficient number of sensors (e.g., 20 sensors). Therefore, minimizing the opportunitydiscovery delay is important not only to help the vacated SUs resume their operation but also to minimize interruption of SUs in other in-band channels.

\subsection{Notation Table}

We summarize the frequently used notations in Table 1.

\section{Sequential Sensing Mechanism}

This section overviews our opportunity discovery mechanism via sequential sensing, to enhance the understanding of the proposed schemes in the later sections.

\subsection{Opportunity Discovery Procedure}

Let $B_{r e q}$ denote the total amount of bandwidth a CRN requires, which is the sum of spectrum demands of all SUs 
TABLE 1

\section{Summary of Notations}

\begin{tabular}{|c|l|}
\hline$M$ & number of licensed channels \\
\hline$N$ & number of backup channels $(N \leq M)$ \\
\hline$i$ & channel index \\
\hline$Z^{i}(t) \in\{0,1\}$ & channel state at time $t(0: \mathrm{OFF}, 1: \mathrm{ON})$ \\
\hline$u^{i} \in[0,1]$ & channel utilization factor \\
\hline$T_{I}^{i}$ & sensing-time \\
\hline$C_{i}$ & channel capacity \\
\hline$\theta_{i}$ & channel idle probability at opportunity discovery \\
\hline
\end{tabular}

in the CRN. ${ }^{2} B_{r e q}$ may be achieved by utilizing just one idle channel with $C_{i} \geq B_{\text {req }}$ or by simultaneously utilizing multiple idle channels whose combined capacity exceeds $B_{r e q}$. Therefore, a CRN utilizes one or more in-band channels to achieve $B_{\text {req }}$.

Opportunity discovery is triggered when one of the inband channels is vacated due to the returning PUs. At opportunity discovery, a CRN needs to find a set of new idle channels whose collective capacity achieves $B_{\text {target }}$, which is given as $B_{\text {target }}=\max \left\{B_{\text {req }}-B_{\text {in-band }}, 0\right\}$, where $B_{\text {in-band }}$ is the sum capacity of the remaining in-band channels after the channel vacation.

New idle channels are discovered by sequentially sensing backup channels such that SUs synchronously tune to one backup channel at a time following a given sensing sequence. Once a backup channel is detected idle, it becomes an in-band channel. The CRN tracks the combined capacity of all current in-band channels, and opportunity discovery completes when the capacity reaches or exceeds $B_{\text {req }}$.

Fig. 3 shows an example where all four channels have the same capacity and the CRN requires two idle channels to meet its $B_{\text {req }}$. In the figure, the delay of each opportunity discovery is also shown, which is the sum of sensing-times spent to sequentially sense the backup channels until $B_{\text {req }}$ is achieved. Although in this example it suffices to find one additional idle channel per discovery, we will consider general scenarios in the next section, where achieving $B_{r e q}$ may require to find more than one idle channel.

\subsection{Channel Idle Probability Prediction}

In this paper, we characterize a channel by a tuple of $\left\{T_{I}^{i}, C_{i}, \theta_{i}\right\}$, where $T_{I}^{i}$ is the sensing time, $C_{i}$ the channel capacity, and $\theta_{i}$ the probability that channel $i$ would be idle if the channel is sensed at opportunity discovery. Although derivation of $\theta_{i}$ for alternating renewal channels is described in [2], we briefly overview the procedure for completeness of the presentation.

$\theta_{i}$ varies with channels and time because it depends on the ON/OFF usage pattern and the history of sensing results (or samples). Therefore, calculation of $\theta_{i}$ first requires estimation of channel parameters, e.g., $\psi_{O F F}^{i}$ and $\psi_{O N}^{i}$ in (1). In [2], an ML estimator and its confidence interval was derived when a set of samples from channel $i$ is given as $\mathrm{Z}^{i}=\left(Z^{i}\left(t_{1}\right), Z^{i}\left(t_{2}\right), \ldots, Z^{i}\left(t_{r}\right)\right)$ where $t_{1}<t_{2}<\cdots<t_{r}$. The $r$ samples can be collected at any time, i.e., they are not necessarily periodic, because they are produced by:

2. We consider a persistent transmission model, where each SU continuously transmits at a constant rate in its in-band channel. Hence, $B_{\text {req }}$ is interpreted as the sum of the rates of all SUs in the CRN.

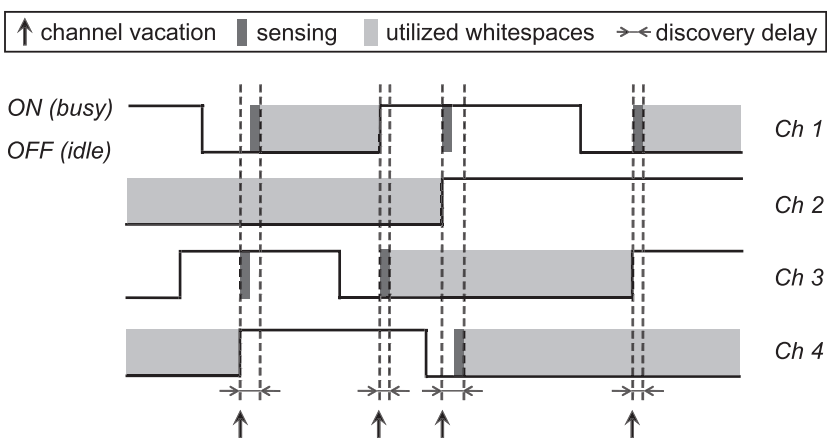

Fig. 3. An illustration of opportunity discovery when a CRN requires two idle channels for its operation.

1) sequential sensing at opportunity discovery (if the channel is selected and sensed); and/or 2) extra sampling scheduled by the central controller who assigns some idle SUs (i.e., SUs with no transmission) to sense out-of-band channels (even when no opportunity discovery is necessary). The extra sampling is to enhance the accuracy of estimation but is outside of the scope of this paper.

Then, [2] showed that $\theta_{i}$ of the renewal processes at the opportunity discovery time $t$ is given as

$$
\begin{aligned}
\theta_{i} & =\operatorname{Pr}\left(Z^{i}(t)=0 \mid Z^{i}\left(t_{1}\right), \ldots, Z^{i}\left(t_{r}\right)\right) \\
& =\operatorname{Pr}\left(Z^{i}(t)=0 \mid Z^{i}\left(t_{r}\right)\right),
\end{aligned}
$$

where $\operatorname{Pr}\left(Z^{i}(t)=0 \mid Z^{i}\left(t_{r}\right)\right)$ is the transition probability between the most recent sample at time $t_{r}$ and an imaginary sample 0 at time $t$. The transition probability is expressed with the estimated channel parameters. For example, exponentially distributed ON/OFF periods lead to the following [2]:

$$
\theta_{i}= \begin{cases}\left(1-u^{i}\right)+u^{i} \cdot e^{-\left(\psi_{O F F}^{i}+\psi_{O N}^{i}\right)\left(t-t_{r}\right)}, & \text { if } Z^{i}\left(t_{r}\right)=0, \\ \left(1-u^{i}\right)\left\{1-e^{-\left(\psi_{O F F}^{i}+\psi_{O N}^{i}\right)\left(t-t_{r}\right)}\right\}, & \text { otherwise }\end{cases}
$$

where $\psi_{O N}^{i}$ and $\psi_{O F F}^{i}$ are from (1).

One can notice that $\theta_{i} \neq\left(1-u^{i}\right)$ because $u^{i}$ is a long-term average utilization of channel $i$ while $\theta_{i}$ is the instantaneous channel idle probability considering the past channel samples. This also implies that $\theta_{i}$ reflects the correlation between the samples. For example, if $Z^{i}\left(t_{r}\right)=0$ and $\left(t-t_{r}\right)$ is small, it is likely that the channel is still in its OFF state, thus making $\theta_{i}$ very close to 1 . On the contrary, if $Z^{i}\left(t_{r}\right)=1$ and $\left(t-t_{r}\right)$ is small, it is likely that the channel is still in its ON state, thus making $\theta_{i}$ very close to 0 . In addition, once a channel with a long ON period enters the ON state, the channel's $\theta_{i}$ becomes very small until enough time passes.

\subsubsection{Sensing Error Compensation}

Although the derivation of $\theta_{i}$ is exact, its correctness relies on how accurately the samples reflect the actual channel state. In reality, spectrum sensing is imperfect since $P M D$ and $P F A$ are nonzero. Since the impact of imperfect sensing on $\theta_{i}$ was not considered in [2], here we propose Bayesian state estimation [27] that can compensate for the sensing error. For the ease of notation, we omit $i$ in this section.

Suppose $\tilde{Z}\left(t_{k}\right) \in\{0,1\}$ denotes the actual state of a channel at time $t_{k}$. Assuming the pdf of the initial state $\tilde{Z}\left(t_{0}\right)$ is known, the estimator is 


$$
f\left(\tilde{Z}\left(t_{0}\right) \mid \mathbf{Z}_{0}\right)=f\left(\tilde{Z}\left(t_{0}\right)\right)
$$

where $\mathbf{Z}_{k}=\left\{Z\left(t_{1}\right), Z\left(t_{2}\right), \ldots, Z\left(t_{k}\right)\right\}$ for $k \geq 1$ and $\mathbf{Z}_{0}=\emptyset$. For each $k \geq 1$, we evaluate

$$
\begin{aligned}
f\left(\tilde{Z}\left(t_{k}\right) \mid \mathbf{Z}_{k-1}\right) & =\sum_{\tilde{Z}\left(t_{k-1}\right)} f\left(\tilde{Z}\left(t_{k}\right) \mid \tilde{Z}\left(t_{k-1}\right)\right) f\left(\tilde{Z}\left(t_{k-1}\right) \mid \mathbf{Z}_{k-1}\right), \\
f\left(\tilde{Z}\left(t_{k}\right) \mid \mathbf{Z}_{k}\right) & =\frac{f\left(Z\left(t_{k}\right) \mid \tilde{Z}\left(t_{k}\right)\right) f\left(\tilde{Z}\left(t_{k}\right) \mid \mathbf{Z}_{k-1}\right)}{\sum_{\tilde{Z}\left(t_{k}\right)} f\left(Z\left(t_{k}\right) \mid \tilde{Z}\left(t_{k}\right)\right) f\left(\tilde{Z}\left(t_{k}\right) \mid \mathbf{Z}_{k-1}\right)},
\end{aligned}
$$

where $f\left(\tilde{Z}\left(t_{k}\right) \mid \mathbf{Z}_{k-1}\right)$ is the prior probability mass function (pmf) of $\tilde{Z}\left(t_{k}\right)$ before observing $Z\left(t_{k}\right)$ and $f\left(\tilde{Z}\left(t_{k}\right) \mid \mathbf{Z}_{k}\right)$ is the posterior pmf of $\tilde{Z}\left(t_{k}\right)$ after observing $Z\left(t_{k}\right)$.

Prior and posterior pmfs are updated whenever a new sample $Z\left(t_{k}\right)$ is obtained. Then, when opportunity discovery is triggered at time $t, \theta$ can be estimated as

$$
\theta=\left.f\left(\tilde{Z}\left(t_{k}\right) \mid \mathbf{Z}_{k-1}\right)\right|_{t_{k}=t, \tilde{Z}\left(t_{k}\right)=0} .
$$

In the above procedure, $f\left(\tilde{Z}\left(t_{k}\right) \mid \tilde{Z}\left(t_{k-1}\right)\right)$ and $f\left(Z\left(t_{k}\right) \mid\right.$ $\left.\tilde{Z}\left(t_{k}\right)\right)$ are yet to be determined. $f\left(\tilde{Z}\left(t_{k}\right) \mid \tilde{Z}\left(t_{k-1}\right)\right)$ is the transition probability between two consecutive samples at times $t_{k-1}$ and $t_{k}$, which has been fully derived in [2]. On the other hand, $f\left(Z\left(t_{k}\right) \mid \tilde{Z}\left(t_{k}\right)\right)$ is easily determined according to the definition of $P M D$ and $P F A$ such as

$$
f\left(Z\left(t_{k}\right) \mid \tilde{Z}\left(t_{k}\right)\right)= \begin{cases}1-P F A, & \text { if }\left(\tilde{Z}\left(t_{k}\right), Z\left(t_{k}\right)\right)=(0,0) \\ P F A, & \text { if }\left(\tilde{Z}\left(t_{k}\right), Z\left(t_{k}\right)\right)=(0,1) \\ 1-P M D, & \text { if }\left(\tilde{Z}\left(t_{k}\right), Z\left(t_{k}\right)\right)=(1,0) \\ P M D, & \text { if }\left(\tilde{Z}\left(t_{k}\right), Z\left(t_{k}\right)\right)=(1,1) .\end{cases}
$$

\section{Optimal Sensing Sequence for Minimal OPPORTUNITY-DISCOVERY LATENCY}

In this section, we derive an optimal sensing sequence of backup channels that incurs the minimal delay in discovering a necessary amount of opportunities and propose a computationally efficient sequence algorithm that provides a near-optimal performance. As introduced in Section 4, backup channels are characterized by a tuple of $\left\{T_{I}^{i}, C_{i}, \theta_{i}\right\}$ and the opportunity discovery is triggered at channel vacation of an in-band channels, where the target amount of bandwidth to discover is denoted by $B_{\text {target }}$. For the notational ease, we will use $B$ to denote $B_{\text {target }}$ throughout this section.

\subsection{Problem Statement}

Suppose there are $N(\leq M)$ backup channels, and let $S=$ $\left\{s_{1}, s_{2}, \ldots, s_{N}\right\} \in \mathcal{S}$ be an ordered list of $N$ channels, where $s_{j}$ is the channel index of the $j$ th channel in the sequence $\left(s_{j}\right.$ : positive integer, $\left.1 \leq s_{j} \leq N\right)$ and $\mathcal{S}$ is the set of all possible channel sequences $\left(|\mathcal{S}|=N\right.$ !). Also suppose $T_{I}^{i}, C_{i}$, and $\theta_{i}$ are known a priori.

Our objective is to determine the optimal sensingsequence $S^{*}$ that minimizes the average delay in finding idle channels whose cumulative capacity exceeds $B$. This can be stated formally as:

Find $S^{*}=\underset{S \in \mathcal{S}}{\arg \min } E_{\boldsymbol{\theta}}\left[\sum_{i=1}^{N} T_{I}^{s_{i}} \cdot I_{\left\{\sum_{j=1}^{i-1} C_{s_{j}} \cdot\left(1-Z^{s_{j}}\right)<B\right\}}\right]$, where

$$
\begin{aligned}
\boldsymbol{\theta} & =\left(\theta_{1}, \theta_{2}, \ldots, \theta_{N}\right), \\
I_{\{\star\}} & = \begin{cases}1, & \text { if the statement } \star \text { is true }, \\
0, & \text { otherwise. }\end{cases}
\end{aligned}
$$

In the above problem statement, the indicator function

$$
I_{\left\{\sum_{j=1}^{i-1} C_{s_{j}} \cdot\left(1-Z^{s_{j}}\right)<B\right\}}
$$

implies that once $B$ is achieved, the sequential sensing stops. $^{3}$

\subsection{Offline versus Online Sensing Sequences}

To find the optimal sensing sequence, we need to consider two types of sensing sequence: Offline and online sequences. In what follows, we will introduce the concept of the two sequences and discuss the reason why the online sequence must be considered to find the optimal sensing sequence.

\subsubsection{Offline Sequence}

An offline sensing sequence is a static sequence, which strictly follows the initially determined sequence regardless of the channel states observed during the sequential sensing. The optimal offline sensing sequence is built by considering $\theta$ while ignoring the actual channel states to be observed, and the average discovery delay $\mathcal{D}$ of a given offline sequence $S=\left\{s_{1}, s_{2}, \ldots, s_{N}\right\}$ is given as

$$
\begin{aligned}
\mathcal{D}= & \sum_{z_{1}=0}^{1} \sum_{z_{2}=0}^{1} \ldots \sum_{z_{N}=0}^{1}\left\{\prod_{v=1}^{N}\left(\theta_{s_{v}}\right)^{1-z_{v}}\left(1-\theta_{s_{v}}\right)^{z_{v}}\right. \\
& \left.\times\left(T_{I}^{s_{1}}+\sum_{v=2}^{N} T_{I}^{S_{v}} \cdot I_{\left\{\sum_{w=1}^{v-1} C_{s_{w}}\left(1-z_{w}\right)<B\right\}}\right)\right\} .
\end{aligned}
$$

Then, the optimal offline sequence is determined as the sequence that minimizes (3) among $N$ ! possible sequences.

As an example, let us consider the following scenario:

$$
\begin{aligned}
& N=3, \quad B=2.0, \quad\left(C_{1}, C_{2}, C_{3}\right)=(0.5,1.5,2.0), \\
& \left(T_{I}^{1}, T_{I}^{2}, T_{I}^{3}\right)=(1,2,3), \quad\left(\theta_{1}, \theta_{2}, \theta_{3}\right)=(0.5,0.3,0.1) .
\end{aligned}
$$

By defining $\tilde{\theta}_{s_{j}}:=1-\theta_{s_{j}}$, (3) becomes

$$
\begin{aligned}
\mathcal{D}= & \tilde{\theta}_{s_{1}} \tilde{\theta}_{s_{2}} \tilde{\theta}_{s_{3}}\left(T_{I}^{s_{1}}+T_{I}^{s_{2}}+T_{I}^{s_{3}}\right) \\
& +\tilde{\theta}_{s_{1}} \tilde{\theta}_{s_{2}} \theta_{s_{3}}\left(T_{I}^{s_{1}}+T_{I}^{s_{2}}+T_{I}^{s_{3}}\right) \\
& +\tilde{\theta}_{s_{1}} \theta_{s_{2}} \tilde{\theta}_{s_{3}}\left(T_{I}^{s_{1}}+T_{I}^{s_{2}}+T_{I}^{s_{3}} \cdot I_{\left\{C_{s_{2}}<B\right\}}\right) \\
& +\tilde{\theta}_{s_{1}} \theta_{s_{2}} \theta_{s_{3}}\left(T_{I}^{s_{1}}+T_{I}^{s_{2}}+T_{I}^{s_{3}} \cdot I_{\left\{C_{s_{2}}<B\right\}}\right) \\
& +\theta_{s_{1}} \tilde{\theta}_{s_{2}} \tilde{\theta}_{s_{3}}\left(T_{I}^{s_{1}}+T_{I}^{s_{2}} \cdot I_{\left\{C_{s_{1}}<B\right\}}+T_{I}^{s_{3}} \cdot I_{\left\{C_{s_{1}}<B\right\}}\right) \\
& +\theta_{s_{1}} \tilde{\theta}_{s_{2}} \theta_{s_{3}}\left(T_{I}^{s_{1}}+T_{I}^{s_{2}} \cdot I_{\left\{C_{s_{1}}<B\right\}}+T_{I}^{s_{3}} \cdot I_{\left\{C_{s_{1}}<B\right\}}\right) \\
& +\theta_{s_{1}} \theta_{s_{2}} \tilde{\theta}_{s_{3}}\left(T_{I}^{s_{1}}+T_{I}^{s_{2}} \cdot I_{\left\{C_{s_{1}}<B\right\}}+T_{I}^{s_{3}} \cdot I_{\left\{C_{s_{1}}+C_{s_{2}}<B\right\}}\right) \\
& +\theta_{s_{1}} \theta_{s_{2}} \theta_{s_{3}}\left(T_{I}^{s_{1}}+T_{I}^{s_{2}} \cdot I_{\left\{C_{s_{1}}<B\right\}}+T_{I}^{s_{3}} \cdot I_{\left\{C_{s_{1}}+C_{s_{2}}<B\right\}}\right) .
\end{aligned}
$$

3. In practice, $\theta$ must be updated after sensing each channel by recalculating $\theta_{i}$ s based on the elapsed time due to sensing of the last chosen channel. Although updating $\theta$ is always possible by following the procedure in [2], here we assume $T_{I}^{i} \mathrm{~s}$ are small enough to make the impact of adjusting $\theta_{i}$ 's negligible, for the purpose of easier illustration. In fact, this assumption is applicable in most practical scenarios because $T_{I}^{i}$ is usually in the order of milliseconds (or even less) while the ON/OFF periods are typically in the order of seconds or minutes. 
Then, by comparing all 3 ! sequences, the optimal offline sequence that minimizes (5) is found to be $\{1,2,3\}$.

\subsubsection{Online Sequence}

An online sensing sequence, on the other hand, is a dynamic sequence that can update itself each time it sense a channel in the sequence such that the remaining unsensed channels are reordered to form an updated subsequence according to the observed channel states. Such a dynamic sequence is necessary to derive the optimal sensing sequence because the optimality condition changes whenever we sense a new channel and observe its actual state.

To show the impact of online observation of channel states, let us consider again the example in (4). Once the first channel in the optimal offline sequence (i.e., channel 1) is sensed, the capacity-to-go (i.e., the remaining capacity to achieve at the subsequent sensing) is updated as

$$
B^{\prime}= \begin{cases}B-C_{s_{1}}, & \text { if channel } 1 \text { is sensed idle, } \\ B, & \text { otherwise. }\end{cases}
$$

Then, we need to find the best channel to sense next to minimize the subsequent sensing delay $\mathcal{D}^{\prime}$ in sensing $S^{\prime}=\left\{s_{2}, s_{3}\right\}$, where

$$
\begin{aligned}
\mathcal{D}^{\prime}= & \tilde{\theta}_{s_{2}} \tilde{\theta}_{s_{3}}\left(T_{I}^{s_{2}}+T_{I}^{s_{3}}\right)+\tilde{\theta}_{s_{2}} \theta_{s_{3}}\left(T_{I}^{s_{2}}+T_{I}^{s_{3}}\right) \\
& +\theta_{s_{2}} \tilde{\theta}_{s_{3}}\left(T_{I}^{s_{2}}+T_{I}^{s_{3}} \cdot I_{\left\{C_{s_{2}}<B^{\prime}\right\}}\right) \\
& +\theta_{s_{2}} \theta_{s_{3}}\left(T_{I}^{s_{2}}+T_{I}^{s_{3}} \cdot I_{\left\{C_{s_{2}}<B^{\prime}\right\}}\right) .
\end{aligned}
$$

Using $B^{\prime}$ and $\mathcal{D}^{\prime}$ and comparing all $(N-1)$ ! possible subsequences, it can be seen that the optimal choice of $s_{2}$ minimizing $\mathcal{D}^{\prime}$ is given as

$$
s_{2}= \begin{cases}2, & \text { if channel } 1 \text { is sensed idle, } \\ 3, & \text { otherwise. }\end{cases}
$$

Therefore, the optimal offline sequence $\{1,2,3\}$ is no longer optimal if the first channel is sensed busy. Intuitively speaking, when channel 1 is idle, we have $B^{\prime}=1.5$, and thus, we may still want to sense channel 2 next because $C_{2}=1.5$ is large enough to fulfill $B^{\prime}$. When channel 1 is busy, however, we have $B^{\prime}=2.0$ and we may want to sense channel 3 instead since channel 2 cannot fulfill $B^{\prime}$ while channel 3 can (since $C_{3}=2.0$ ). That is, if $s_{2}=2$, the subsequent delay always becomes $\left(T_{I}^{2}+T_{I}^{3}\right)$ regardless of the state of channel 2, whereas the delay might become $T_{I}^{3}$ if $s_{2}=3$ and channel 3 is sensed idle.

\subsection{Optimal Online Sensing Sequence Algorithm}

As shown in Section 5.2, we need to find the optimal online sequence to minimize the opportunity discovery delay. To find the optimal online sequence, we propose a dynamicprogramming-based search algorithm. We will also show the optimal sequence takes a simple form for the special case of homogeneous channel capacities.

Let us define an $N$-stage decision problem, where at stage $k$ we have $(N-k+1)$ channels to choose from and $(k-1)$ channels already sensed. Our objective is then, at stage $k$, to make an optimal decision on which channel to sense next, to minimize the overall delay in achieving the target amount of bandwidth, based on the alreadydiscovered idle channels. For this, we define control $u_{k}$ as the chosen channel to sense at stage $k$. We also define $x_{k}=$ $\left(U_{k}, B_{k}\right)$ as the state at stage $k$, where $U_{k}$ is the set of remaining channels to choose from and $B_{k}$ is the accumulated bandwidth achieved from the idle channels among the already sensed. Finally, we define $g_{k}\left(x_{k}, u_{k}\right)$ as the cost incurred at stage $k$ by the chosen control $u_{k}$, which is in fact the sensing time $T_{I}^{u_{k}}$ of the chosen channel $u_{k}$ because our goal is to minimize the overall sensing time.

Now, the DP algorithm for the optimal online sequence is formulated as follows:

$$
\begin{aligned}
J_{N}\left(x_{N}\right) & =g_{N}\left(x_{N}\right), \\
J_{k}\left(x_{k}\right) & =\min _{u_{k} \in U_{k}}\left\{E_{\boldsymbol{\theta}}\left[g_{k}\left(x_{k}, u_{k}\right)+J_{k+1}\left(x_{k+1}\right)\right]\right\}, \\
u_{k}\left(x_{k}\right) & =\arg \min _{u \in U_{k}}\left\{E_{\boldsymbol{\theta}}\left[g_{k}\left(x_{k}, u\right)+J_{k+1}\left(x_{k+1}\right)\right]\right\},
\end{aligned}
$$

where $g_{k}\left(x_{k}, u_{k}\right)=T_{I}^{u_{k}} \cdot I_{\left\{B_{k}<B\right\}}$. Then, (7) can be further analyzed using

$$
\begin{aligned}
& U_{k+1}=U_{k} \backslash\left\{u_{k}\right\}, \\
& B_{k+1}= \begin{cases}B_{k}+C_{u_{k}}, & \text { if channel } u_{k} \text { is idle, } \\
B_{k}, & \text { otherwise, }\end{cases}
\end{aligned}
$$

such that

$$
\begin{aligned}
J_{k}\left(x_{k}\right)= & \min _{u_{k} \in U_{k}}\left\{T_{I}^{u_{k}} \cdot I_{B_{k}<B}+\theta_{u_{k}} \cdot J_{k+1}\left(U_{k+1}, B_{k}+C_{u_{k}}\right)\right. \\
& \left.+\left(1-\theta_{u_{k}}\right) \cdot J_{k+1}\left(U_{k+1}, B_{k}\right)\right\} .
\end{aligned}
$$

As an initial condition, we have

$$
U_{1}=\{1,2, \ldots, N\}, \quad B_{1}=0 .
$$

Fig. 4 presents the pseudocode of the proposed DP algorithm.

\subsubsection{Algorithm Complexity Analysis}

Once $J_{N}\left(x_{N}\right)$ is computed for every possible $x_{N}$ 's, $J_{k}\left(x_{k}\right)$ for $k \leq N-1$ can be iteratively derived. Therefore, the complexity of the algorithm depends on the number of possible combinations of $U_{N}$ and $B_{N}$. Since $U_{N}$ is a set of a single element, there are $N$ possible choices of $U_{N}$. For a given $U_{N}$ $B_{N}$ is determined by considering all possible combinations of the channel states (i.e., idle or busy) of the preceding $N-1$ channels-that is, $2^{N-1}$. As a result, there are $N \cdot 2^{N-1}$ possible choices of $x_{N}=\left(U_{N}, B_{N}\right)$ which gives us the complexity of $O\left(N \cdot 2^{N}\right)$, and hence, the DP algorithm is not a polynomial-time solution. In Section 5.4, we propose a computationally efficient suboptimal algorithm that yields a near-optimal performance.

\subsubsection{A Special Case: Homogeneous Channel Capacity}

In this section, we will prove that in a special case with homogeneous channel capacities, i.e., $C_{i}=C, \forall i$, the optimal online sensing-sequence takes a much simpler form. In Lemma 1, we first prove that the optimal offline sensing sequence takes a trivial form with $C_{i}=C$, and then in Theorem 1 we prove that the optimal offline sequence becomes the optimal online sequence under the same condition.

Lemma 1. If $C_{i}=C, \forall i$, then the optimal offline sensing-sequence is built by sorting channels in ascending order of $T_{I}^{i} / \theta_{i}$. 
$B:=$ the total amount of bandwidth to discover;

$U_{1}:=\{1,2, \ldots, N\}, \quad B_{1}:=0, \quad k:=1$;

For all possible combinations of $x_{N}=\left(U_{N}, B_{N}\right)$, derive $J_{N}\left(x_{N}\right)$ and $u_{N}\left(x_{N}\right)$ by Eq. (7). Then, using $J_{N}\left(x_{N}\right)$, derive all preceding $J_{k}\left(x_{k}\right)$ and $u_{k}\left(x_{k}\right)$ according to Eq. (7), for $k=N-1, N-2, \ldots, 1$;

while $(k \leq N)\{$

$S_{k}^{*}:=u_{k}\left(x_{k}\right)$;

Sensing sequence $S^{*}:=\left\{S_{1}^{*}, S_{2}^{*}, \ldots, S_{k}^{*}\right\}$;

Sense $S_{k}^{*}$ and discover its channel state $Z$; if $(Z==0)$ then

$\begin{aligned} B_{k+1} & :=B_{k}+C_{S_{k}^{*}} ; \\ \text { else } \quad B_{k+1} & :=B_{k}\end{aligned}$

if $\left(B_{k+1} \geq B\right)$ then

Stop the sequential sensing;

return;

$U_{k+1}:=U_{k} \backslash\left\{S_{k}^{*}\right\} ;$

$k:=k+1$;

\}

return;

Fig. 4. Pseudocode of the DP search algorithm ( $S^{*}$ : optimal sequence).

Proof. Let $L$ be the optimal sensing sequence and $L^{\prime}$ be its counterpart constructed by switching the order of the $k$ th and $(k+1)$ th channels in $L$. That is,

$$
\begin{aligned}
L & =\left(l_{1}, \ldots, l_{k-1}, l_{k}, l_{k+1}, l_{k+2}, \ldots, l_{N}\right), \\
L^{\prime} & =\left(l_{1}, \ldots, l_{k-1}, l_{k+1}, l_{k}, l_{k+2}, \ldots, l_{N}\right) .
\end{aligned}
$$

On the other hand, $\mathcal{D}_{L}^{B}$ is defined as the average delay in locating idle channels whose cumulative capacity exceeds $B$, using a sensing-sequence $L . \mathcal{P}_{L}^{B}$ is defined as the probability that the sum of capacities of idle channels in a sensing-sequence $L$ may be strictly less than $B$.

In addition, let us define the following ordered lists:

$$
\begin{aligned}
L_{k-1} & =\left(l_{1}, l_{2}, \ldots, l_{k-1}\right), \quad L_{k}=\left(l_{1}, l_{2}, \ldots, l_{k}\right), \\
L_{k+1} & =\left(l_{1}, l_{2}, \ldots, l_{k+1}\right), \quad L_{k+1}^{c}=\left(l_{k+2}, \ldots, l_{N}\right), \\
L_{k-1, k+1} & =\left(l_{1}, l_{2}, \ldots, l_{k-1}, l_{k+1}\right), \\
L_{k-1, k+1, k} & =\left(l_{1}, l_{2}, \ldots, l_{k-1}, l_{k+1}, l_{k}\right) .
\end{aligned}
$$

Since a channel is sensed only when those channels preceding in the list provide less opportunities than $B$, we can express $\mathcal{D}_{L}^{B}$ and $\mathcal{D}_{L^{\prime}}^{B}$ as

$$
\begin{aligned}
\mathcal{D}_{L}^{B}= & \mathcal{D}_{L_{k-1}}^{B}+\mathcal{P}_{L_{k-1}}^{B} \cdot T_{I}^{l_{k}}+\mathcal{P}_{L_{k}}^{B} \cdot T_{I}^{l_{k+1}}+\mathcal{P}_{L_{k+1}}^{B} \cdot \mathcal{D}_{L_{k+1}^{c}}^{B}, \\
\mathcal{D}_{L^{\prime}}^{B}= & \mathcal{D}_{L_{k-1}}^{B}+\mathcal{P}_{L_{k-1}}^{B} \cdot T_{I}^{l_{k+1}}+\mathcal{P}_{L_{k-1, k+1}}^{B} \cdot T_{I}^{l_{k}} \\
& +\mathcal{P}_{L_{k-1, k+1, k}}^{B} \cdot \mathcal{D}_{L_{k+1}^{c}}^{B} \cdot
\end{aligned}
$$

Since $\mathcal{D}_{L}^{B} \leq \mathcal{D}_{L^{\prime}}^{B}$ and $\mathcal{P}_{L_{k+1}}^{B}=\mathcal{P}_{L_{k-1, k+1, k}}^{B}$, we have

$$
\mathcal{P}_{L_{k-1}}^{B} \cdot T_{I}^{l_{k}}+\mathcal{P}_{L_{k}}^{B} \cdot T_{I}^{l_{k+1}} \leq \mathcal{P}_{L_{k-1}}^{B} \cdot T_{I}^{l_{k+1}}+\mathcal{P}_{L_{k-1, k+1}}^{B} \cdot T_{I}^{l_{k}},
$$

which reduces to

$$
\frac{T_{I}^{l_{k}}}{\left(\mathcal{P}_{L_{k-1}}^{B}-\mathcal{P}_{L_{k-1}}^{B-C_{l_{k}}}\right) \theta_{l_{k}}} \leq \frac{T_{I}^{l_{k+1}}}{\left(\mathcal{P}_{L_{k-1}}^{B}-\mathcal{P}_{L_{k-1}}^{B-C_{k+1}}\right) \theta_{l_{k+1}}},
$$

because $\mathcal{P}_{L_{k}}^{B}=\mathcal{P}_{L_{k-1}}^{B} \cdot\left(1-\theta_{l_{k}}\right)+\mathcal{P}_{L_{k-1}}^{B-C_{l_{k}}} \cdot \theta_{l_{k}}$.

By substituting $C$ for $C_{l_{k}}$ and $C_{l_{k+1}}$ in (9), the inequality condition reduces to:

$$
T_{I}^{l_{k}} / \theta_{l_{k}} \leq T_{I}^{l_{k+1}} / \theta_{l_{k+1}}, \text { for } 1 \leq k \leq N-1,
$$

which is a necessary condition for optimality. However, because there exists a single and unique sequence satisfying such a necessary condition, ${ }^{4}$ the condition also becomes sufficient. Therefore, the resulting sequence is optimal.

Now, using Lemma 1, we present the following theorem on the optimal online sensing sequence.

Theorem 1. If $C_{i}=C, \forall i$, then the optimal online sensing sequence is built in the same way as the optimal offline sensing sequence, by sorting channels in ascending order of $T_{I}^{i} / \theta_{i}$.

Proof. The optimal online sequence is built by repeatedly searching for the optimal offline subsequences. Let us denote by $S$ the optimal offline sequence of all $N$ channels. According to Lemma $1, S$ is simply constructed by sorting channels in ascending order of $T_{I}^{i} / \theta_{i}$. Then, the first channel in $S$, say $s$, becomes the first channel in the optimal online sequence. Now, to find the next channel in the optimal online sequence, we need to sort $S \backslash\{s\}$, again, according to Lemma 1 . However, since (10) does not depend on $B$, the sorted sequence of $S \backslash\{s\}$ is still the same as $S \backslash\{s\}$. Therefore, the second channel in the optimal online sequence is the second entry of $S$. By the same argument, $S$ becomes the optimal online sequence, which proves the theorem.

\subsection{Suboptimal Sensing Sequence Algorithm}

The proposed DP algorithm suffers from high computational complexity. Therefore, we propose a computationally efficient algorithm that determines a suboptimal sequence in polynomial time while providing a nearoptimal performance.

To derive the suboptimal sequence, we utilize the necessary condition for optimality in (9). We first recognize that the first channel in the sequence (i.e., $k=1$ ) satisfies $L_{k-1}=\emptyset$, and thus, we have $\mathcal{P}_{L_{k-1}}^{B}=1$ and

$$
\mathcal{P}_{L_{k-1}}^{B-C_{l_{k}}}= \begin{cases}0, & \text { if } C_{l_{k}} \geq B, \\ 1, & \text { otherwise, }\end{cases}
$$

by the definition of $\mathcal{P}_{L}^{B}$. ${ }^{5}$ Then, we get

$$
\frac{T_{I}^{l_{k}}}{\left(\mathcal{P}_{L_{k-1}}^{B}-\mathcal{P}_{L_{k-1}}^{B-C_{l_{k}}}\right) \theta_{l_{k}}}= \begin{cases}T_{I}^{l_{k}} / \theta_{l_{k}}, & \text { if } C_{l_{k}} \geq B, \\ \infty, & \text { otherwise. }\end{cases}
$$

4. Note that since $\theta_{i} \in[0,1]$, it is not likely to have ties with the same $T_{I}^{i} / \theta_{i}$. If it happens, we can still sort them uniquely in ascending/ descending order of channel index $i$.

5 . Note that the sum capacity of idle channels in an empty set is equal to zero. 


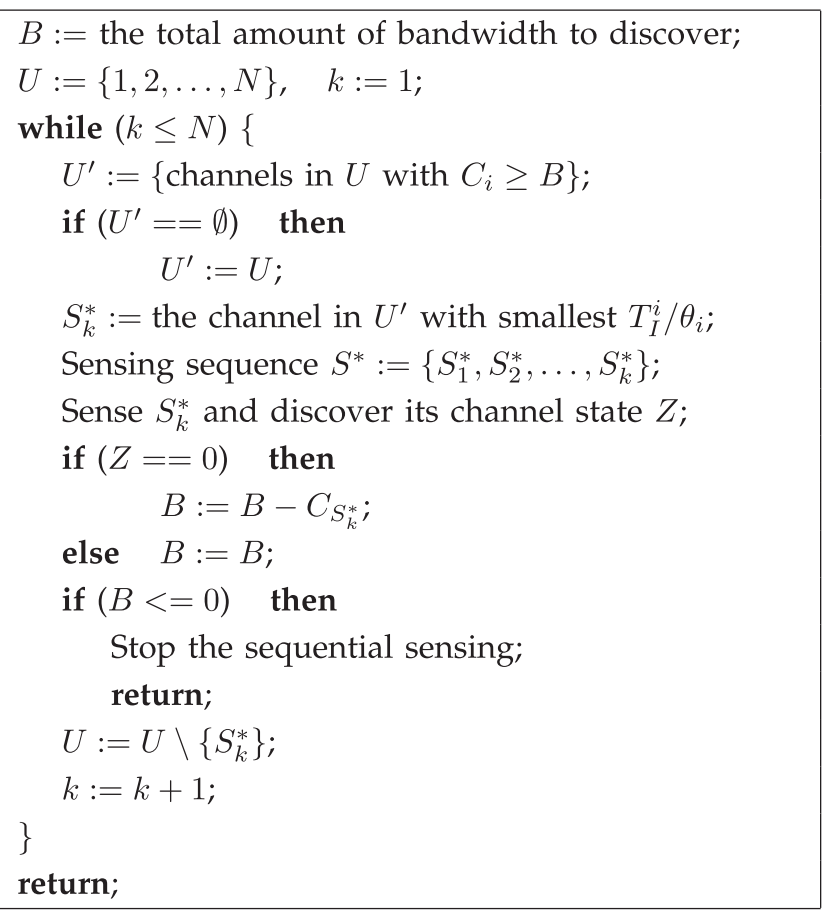

Fig. 5. Pseudocode of the proposed suboptimal sensing sequence algorithm ( $S^{*}$ : near-optimal sequence).

Therefore, to satisfy (9), the first channel should be the one with the smallest $T_{I}^{i} / \theta_{i}$ among all unsensed channels satisfying $C_{i} \geq B$. In case all unsensed channels have $C_{i}<B$, we have $\mathcal{P}_{L_{k-1}}^{B-C_{l_{k}}}=1$, and thus, the term $\mathcal{P}_{L_{k-1}}^{B}-\mathcal{P}_{L_{k-1}}^{B-C_{l_{k}}}$ in (9) cancels out. As a result, the first channel in this case (i.e., $C_{i}<B$ for all unsensed channels) is found as the one with the smallest $T_{I}^{i} / \theta_{i}$.

Once the first channel is determined, we sense the channel and observe its channel state. Depending on the state, we update $B$ as in (6) and find the next channel to sense. Since the problem becomes identical to the initial problem of finding the first channel except the updated $B$ and the set of unsensed channels (i.e., one less unsensed channels by excluding the one just sensed), we can apply the same procedure described above to find the next channel. Finally, the procedure completes when the updated $B$ satisfies $B \leq 0$.

The pseudocode of the proposed suboptimal sequence algorithm is described in Fig. 5. Its near-optimal performance will be shown in Section 8.

\subsubsection{Algorithm Complexity Analysis}

The algorithm sorts $(N-k+1)$ channels at stage $k$, where $k=1,2, \ldots, N$. Therefore, the number of computations required is $N+(N-1)+\cdots+1=N(N+1) / 2$, and thus, we have $O\left(N^{2}\right)$. Therefore, the suboptimal algorithm is solvable in polynomial time, significantly reducing the computational overhead compared to the DP algorithm.

\subsection{Impact of Channel-State Conversion}

During a sequential sensing, it is possible that some inband channels convert their states to "busy," which is defined as channel-state conversion. Channel-state conversion is detected by in-band sensing [5], which is performed independently of out-of-band sensing (footnote 1). Upon detection of such an event, it increases the optimality condition $B$ by $C_{c o n v}$, the capacity of the channel in which the conversion occurs, thus making the previously derived optimal sequence (in Section 5.3) no longer optimal. Therefore, an optimal sequence should be recalculated at the time of each channel-state conversion.

Fortunately, in the case of suboptimal sequence (Section 5.4), it is not necessary to recalculate the entire sensing sequence as in the optimal case. When channelstate conversion occurs, it can be incorporated in the suboptimal algorithm (Fig. 5) by updating $B:=B+C_{\text {conv }}$. Then, the algorithm will correspondingly determine the next best channel to sense, given the increased $B$, instead of finding the entire sensing sequence again. This is because the suboptimal algorithm is independent of the initial $B$, but only depends on the current $B$ at stage $k$.

Since channel-state conversion increases the target bandwidth $B$, it inflates the latency of opportunity discovery. To better understand the inflation effect, we introduce the probability $p_{c o n v}$ that state conversion occurs per sequential sensing:

$$
p_{\text {conv }}=1-\prod_{i \in S_{\text {in }}} p_{O F F}^{i}\left(\tau_{\text {seq }}\right),
$$

where $S_{i n}$ is the set of in-band channels and $p_{O F F}^{i}\left(\tau_{s e q}\right)$ is the probability that in-band channel $i$ stays OFF during the sequential sensing delay of $\tau_{\text {seq }}$, which depends on the pdf of OFF periods. For instance, an exponentially distributed OFF period gives $p_{O F F}^{i}\left(\tau_{s e q}\right)=e^{-\psi_{O F F}^{i} \tau_{s e q}}$.

Assuming $\tau_{s e q}$ represents an average sequential sensing delay without state conversion, the inflated delay due to state conversion is given as

$$
\sum_{i=0}^{\infty}\left(p_{\text {conv }}\right)^{i}\left(1-p_{\text {conv }}\right) \cdot(i+1) \tau_{\text {seq }}=\frac{\tau_{\text {seq }}}{1-p_{\text {conv }}} .
$$

That is, the latency is inflated by a factor of $f_{\text {conv }}=1 /(1-$ $\left.p_{\text {conv }}\right)$. In Section 8, we measure how much the latency is inflated in the tested scenarios and compare it with the derived model $f_{\text {conv }}$.

\subsection{Retry of Sequential Sensing}

A CRN may sometimes fail to find the necessary amount of opportunities (i.e., $B$ ) after searching all $N$ channels. In such a case, the CRN must retry opportunity discovery after waiting for a certain amount of time, and it should keep retrying until enough opportunities are discovered. The reason for waiting is that the channels sensed busy may still be in the same state if the CRN performs an instant retry. We denote the period of such retries by tRETRY, which is a design parameter.

Once the first opportunity discovery fails, the total discovery delay to accomplish $B$ depends more on $t R E T R Y$ due to the subsequent retries. Therefore, it is desirable to have an enough number of "good" channels in BCL to promote successful opportunity discovery at the first trial. The construction of such a BCL will be discussed in Section 6. 


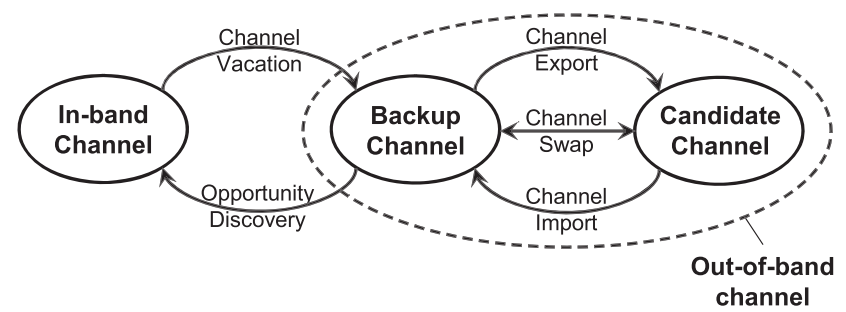

Fig. 6. Transition of channel association.

\section{BCL MANAGEmENT}

The objective of BCL is to maintain "good" channels among the excessively many licensed channels, e.g., $68 \mathrm{TV}$ channels in the VHF/UHF bands [28], to achieve the following goals: 1) Increase the chance of finding spectrum opportunities as much as necessary at each opportunity discovery; and 2) mitigate an overhead in ordering channels by keeping a minimal number of backup channels. To achieve both objectives, we propose a BCL management strategy that constructs and periodically updates the entries of BCL via importing and exporting channels.

Fig. 6 illustrates how a channel changes its association among in-band, backup and candidate channels, according to our BCL management strategy. A backup channel becomes an in-band channel if it is sensed idle during opportunity discovery, and channel vacation of an in-band channel makes it a backup channel again. Channel exports/ imports/swaps are triggered to update the entries of BCL: 1) If the BCL contains backup channels more than necessary, the backup channels with "poor" quality can be exported to CCL; 2) if the BCL needs more channels to provide enough spectrum opportunities, a set of candidate channels can be imported to BCL; and 3) a channel swap exchanges the worst backup channel with the best candidate channel.

\subsection{Construction of Initial BCL}

Initially, we assume there are $M$ licensed channels with no prior knowledge on their channel availability (i.e., $\theta_{i}$ ), because samples are not yet collected from those channels. Without knowing $\theta_{i}$, we construct the initial BCL by randomly selecting $N$ channels from the $M$ channels. From the thus-chosen $N$ channels, the CRN finds in-band channels to start its network with by performing an initial scan. Then, the remaining $(M-N)$ channels are placed in the initial CCL. Note that we define BCL as a combination of in-band channels and (out-of-band) back-up channels, and thus, $N$ will henceforth be used to denote the number of in-band channels plus the number of back-up channels.

One may want to restrict $N$ within some range such as $N_{\text {lower }} \leq N \leq N^{\text {upper }}$, where $N_{\text {lower }}$ and $N_{\text {upper }}$ are design parameters. $N_{\text {lower }}$ helps reserve a minimal number of backup channels so that opportunity discovery may be successful. On the other hand, $N^{\text {upper }}$ upper bounds the computational overhead in sorting backup channels.

\subsection{Periodic BCL Update}

The entries of BCL should keep updated because 1) as sensing accumulates samples of backup channels, ${ }^{6}$ channel

6. As discussed in Section 4.2, the samples are collected via opportunity discovery (if the channel is a backup channel) and/or extra sampling scheduled by the central controller. parameters are estimated more accurately and $\theta_{i}$ can be predicted more reliably, and 2) the parameters of the ON/ OFF distribution may vary with time.

Our goal is to maintain "good" $N$ channels so that they may contain opportunities more than $B_{r e q}$ with probability thPOTENTIAL, which is a predefined threshold (e.g., thPOTENTIAL $=0.9$ ). More formally, we build a sequence of channels $L_{N}=\left\{l_{1}, l_{2}, \ldots, l_{N}\right\}$ where in-band channels are placed first (in any order) and the backup channels are placed last in descending order of $C_{i} \cdot \theta_{i}$ which is called effective capacity of channel $i$. Then, we calculate $\mathcal{C}_{L_{N}}^{B_{\text {req }}}$, capacity potential of $B_{\text {req }}$ in $L_{N}$, representing the probability that $L_{N}$ may contain more opportunities than $B_{\text {req }}$, such that

$$
\begin{aligned}
\mathcal{C}_{L_{N}}^{B_{r e q}}:= & \sum_{z_{1}=0}^{1} \sum_{z_{2}=0}^{1} \ldots \sum_{z_{N}=0}^{1}\left\{\prod_{v=1}^{N}\left(\theta_{l_{v}}\right)^{1-z_{v}}\left(1-\theta_{l_{v}}\right)^{z_{v}}\right. \\
& \left.\times\left(I_{\left\{\sum_{w=1}^{N} C_{l_{w}}\left(1-z_{w}\right) \geq B_{r e q}\right\}}\right)\right\}=1-\mathcal{P}_{L_{N}}^{B_{r e q}} .
\end{aligned}
$$

Using $\mathcal{C}_{L_{N}}^{B_{r e q}}$, we propose an efficient and lightweight BCL update strategy that sorts BCL or CCL separately and only when necessary. In this strategy, BCL is updated periodically every tUPDATE seconds, and at BCL update $\mathcal{C}_{L_{N}}^{B_{\text {req }}}$ is calculated using the most recent channel estimates. According to $\mathcal{C}_{L_{N}}^{B_{\text {req }}}$, one of the following actions is taken: Channel export (BCL $\rightarrow \mathrm{CCL}$ ), channel import $(\mathrm{BCL} \leftarrow \mathrm{CCL})$, and channel swap (BCL $\leftrightarrow \mathrm{CCL})$.

\subsubsection{Channel Export}

If $\mathcal{C}_{L_{N}}^{B_{\text {req }}}>$ thPOTENTIAL $L^{\text {upper }}$, we export a certain number of least preferred channels from BCL because it contains more channels than necessary. We use thPOTENTIAL ${ }^{\text {upper }}=$ thPOTENTIAL $+\epsilon_{1}\left(\epsilon_{1}>0\right)$ to avoid any impetuous channel export. To export channels, we first determine the optimal $N=N^{*}$ such that

$$
N^{*}=\min \left\{N \mid \mathcal{C}_{L_{N}}^{B_{r e q}} \geq \operatorname{thPOTENTIAL}\right\} .
$$

Then, we export the last $\min \left\{N-N^{*}, N_{\text {backup }}\right\}$ channels in the sequence to CCL, where $N_{\text {backup }}$ implies the number of backup channels. Here, the term $N_{\text {backup }}$ is necessary to avoid exporting current in-band channels.

\subsubsection{Channel Import}

If $\mathcal{C}_{L_{N}}^{B_{\text {req }}}<$ thPOTENTIAL $L_{\text {lower, }}$ a number of candidate channels are imported from CCL to satisfy $\mathcal{C}_{L_{N}^{\prime}}^{B_{\text {req }}} \geq$ thPOTENTIAL, where $L_{N}^{\prime}$ is an extended BCL after importing the CCL channels. We use thPOTENTIAL $L_{\text {lower }}=$ thPOTENTIAL $-\epsilon_{2}\left(\epsilon_{2}>0\right)$ to avoid impetuous channel import. To import channels, candidate channels are sorted in descending order of $C_{i} \cdot \theta_{i}$ and are imported to BCL one by one to augment $L_{N}$ by adding the imported channels at the end, until $\mathcal{C}_{L_{N}^{\prime}}^{B_{\text {req }}} \geq \operatorname{thPOTENTIAL}$ is met. In case the imported channel has never been sensed, we assume it has $\theta_{i}=1 / 2$.

\subsubsection{Channel Swap}

When $N_{\text {lower }}$ and $N^{\text {upper }}$ are used, channel export (or import) cannot be processed if $N^{*}=N_{\text {lower }}\left(\right.$ or $N^{\text {upper }}$ ). In such a 
case, we swap the least preferred backup channel with the most preferred candidate channel if the swap helps decrease/increase $\mathcal{C}_{L_{N}}^{B_{\text {req }}}$ as desired.

\section{Channel Parameter Estimation}

In Section 4, we described how to predict $\theta_{i}$, an element indispensable to the formulation of the optimal sensing sequence. There we also showed that $\theta_{i}$ is a function of the $\mathrm{ON} / \mathrm{OFF}$ distribution parameters, and thus, the estimation of the channel parameters' accuracy is a key to the performance of the optimal sensing sequence.

In [2], we introduced an ML estimator of the channel parameters, showing that the sampling period must be proportional to $\min \left\{E\left[T_{O N}^{i}\right], E\left[T_{O F F}^{i}\right]\right\}$ to maintain a similar level of parameter estimation accuracy over channels. To achieve such sampling rates, the central controller of a CRN needs to perform extra sampling on backup channels in addition to the samples produced from opportunity discovery. However, the extra sampling may not be practical for the channels with short ON/OFF periods due to the high-sensing overhead, making the ML estimator an unsuitable choice.

To overcome this problem, we introduce a hybrid of ML and Bayesian estimation. Unlike large-sample asymptotic estimators (e.g., ML) whose estimation accuracy degrades with infrequent samples, Bayesian estimation is known to perform reasonably well even if the number or frequency of samples is limited [7]. Exploiting such features, we propose the following hybrid estimation strategy:

- Class-S channels: Perform Bayesian estimation and

- Class-L channels: Perform ML estimation, where class-S channels imply the channels with short ON/ OFF periods and class- $L$ channels imply the channels with long ON/OFF periods.

In what follows, we first introduce single-step Bayesian inference and its extension to multistage iterative estimation. We will then discuss how to reduce the computational complexity of the Bayesian estimation.

\subsection{Single-Step Bayesian Inference}

A single-step Bayesian inference [7] is summarized as follows: Suppose we have a vector of samples from channel $i$ such as $\mathbf{Z}_{k}^{i}=\left(Z^{i}\left(t_{1}\right), Z^{i}\left(t_{2}\right), \ldots, Z^{i}\left(t_{k}\right)\right)$, whose joint $\mathrm{pmf}$ is $f\left(\mathbf{Z}_{k}^{i} \mid \psi^{i}\right)$ which depends on the vector $\psi^{i} \in \boldsymbol{\Psi}^{i}$ of the channel parameters of $f_{T_{O N}^{i}}(t)$ and $f_{T_{O F F}^{i}}(t)$. Denoting by $\pi\left(\psi^{i}\right)$ a prior distribution of $\psi^{i}$, the posterior distribution of $\psi^{i}$ after observing $\mathbf{Z}_{k}^{i}$, denoted by $\pi\left(\psi^{i} \mid \mathbf{Z}_{k}^{i}\right)$, is given as

$$
\pi\left(\psi^{i} \mid \mathbf{Z}_{k}^{i}\right)=\frac{\pi\left(\psi^{i}\right) f\left(\mathbf{Z}_{k}^{i} \mid \psi^{i}\right)}{f\left(\mathbf{Z}_{k}^{i}\right)}=\frac{\pi\left(\psi^{i}\right) f\left(\mathbf{Z}_{k}^{i} \mid \psi^{i}\right)}{\int_{\Psi^{i}} \pi\left(\psi^{i}\right) f\left(\mathbf{Z}_{k}^{i} \mid \psi^{i}\right) d \psi^{i}},
$$

where $f\left(\mathbf{Z}_{k}^{i}\right)$ is the marginal joint pmf of $\mathbf{Z}_{k}^{i}$. Then, the estimates of $\psi^{i}$ are obtained as

$$
\hat{\psi}^{i}=E\left[\psi^{i}\right],
$$

where $E[\cdot]$ is taken over the distribution $\pi\left(\psi^{i} \mid \mathbf{Z}_{k}^{i}\right)$.

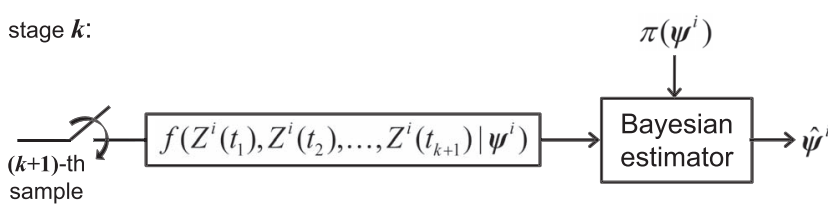

Fig. 7. Iterative Bayesian inference.

\subsection{Iterative Bayesian Inference}

We extend the single-step procedure in Section 7.1 to provide an iterative Bayesian process, where estimates are produced each time a new sample is collected. Fig. 7 illustrates the concept of our iterative Bayesian inference. The process starts with an initial prior distribution $\pi\left(\psi^{i}\right)$, and the first stage begins upon collection of the first two samples. Upon arrival of the $(k+1)$ th sample (i.e., at stage $k$ ), the $k$ th pair of new estimates are computed by using $\pi\left(\psi^{i}\right)$ and $f\left(\mathbf{Z}_{k+1}^{i} \mid \psi^{i}\right)$ of $(k+1)$ samples. We will, henceforth, omit the channel index $i$ unless it causes ambiguity.

For an illustrative purpose, we use exponentially distributed ON and OFF periods with pdfs of (1) to present the proposed procedure. Then, channel parameters to estimate are given as

$$
\psi=\left(\psi_{O N}, \psi_{O F F}\right), \Psi=\{0, \infty\} \times\{0, \infty\} .
$$

It should be noted, however, that the proposed procedure can be applied to $T_{O N}$ and $T_{O F F}$ with general pdfs.

The initial prior distribution $\pi(\psi)$ is usually chosen with subjective reasoning. The criteria in selecting the prior is based on the prior knowledge of $\psi$. For exponentially distributed ON and OFF periods, $\pi(\psi)=\pi\left(\psi_{O N}, \psi_{O F F}\right)$ should be chosen to satisfy the following:

$$
\psi_{O N}>0, \psi_{O F F}>0,
$$

by the definition of exponential distribution. On the other hand, if some statistics are available on average $\mathrm{ON}$ and OFF periods on a large time scale (e.g., a day or a week), such knowledge can be reflected in the choice of the prior. For example, suppose $\tau_{O N}$ and $\tau_{O F F}$ are the average $\mathrm{ON}$ and OFF periods in a day. Then, the prior knowledge can be used to form $\pi(\psi)$ such that

$$
\tau_{O N}=1 / E\left[\psi_{O N}\right], \tau_{O F F}=1 / E\left[\psi_{O F F}\right]
$$

because $\psi_{O N}=1 / E\left[T_{O N}\right]$ and $\psi_{O F F}=1 / E\left[T_{O F F}\right]$.

Here, we assume $\tau_{O N}$ and $\tau_{O F F}$ are given, and the prior distribution is set as

$$
\begin{gathered}
\pi\left(\psi_{O N}, \psi_{O F F}\right)=\tau_{O N} e^{-\tau_{O N} \psi_{O N}} \cdot \tau_{O F F} e^{-\tau_{O F F} \psi_{O F F}} \text { or } \\
\pi\left(u, \psi_{O F F}\right)=\tau_{O N} \tau_{O F F} e^{\left(\tau_{O N}-\tau_{O F F}-\tau_{O N} / u\right) \psi_{O F F}},
\end{gathered}
$$

where $\psi_{O N}$ and $\psi_{O F F}$ are assumed to be exponentially distributed with mean $\tau_{O N}$ and $\tau_{O F F}$, respectively. ${ }^{7}$ Setting the prior distribution as above can satisfy the conditions (12) and (13).

Considering the fact that an alternating renewal process is semi-Markov [22], $f\left(\mathbf{Z}_{k+1} \mid \psi\right)$ at stage $k$ becomes

7. Note that modeling $\psi_{O F F}$ and $\psi_{O N}$ to be exponentially distributed has nothing to do with exponentially distributed ON and OFF periods. 


$$
f\left(\mathbf{Z}_{k+1} \mid \psi\right)=f\left(Z_{t_{1}} \mid \psi\right) f\left(Z_{t_{2}} \mid Z_{t_{1}}, \psi\right) \cdots f\left(Z_{t_{k+1}} \mid Z_{t_{k}}, \psi\right) .
$$

The derivation of the transition probability $f\left(Z_{t_{j+1}} \mid Z_{t_{j}}, \psi\right)$, $j=1,2, \ldots, k$, for arbitrarily-formed $f_{T_{O N}}(t)$ and $f_{T_{O F F}}(t)$ can be found in [22]. For example, with exponentially distributed $\mathrm{ON}$ and OFF periods, we can show

$$
\begin{aligned}
f\left(Z_{t_{1}} \mid \psi\right)= & (1-u)^{1-Z_{t_{1}}} u^{Z_{t_{1}}}, \\
f\left(Z_{t_{j+1}} \mid Z_{t_{j}}, \psi\right)= & (1-u)^{1-Z_{t_{j+1}}} \cdot u^{Z_{t_{j+1}}}+(-1)^{Z_{t_{j}}+Z_{t_{j+1}}} . \\
& u^{1-Z_{t_{j}}} \cdot(1-u)^{Z_{t_{j}}} \cdot e^{-\psi_{O F F} \Delta_{j} / u},
\end{aligned}
$$

where $\Delta_{j}=t_{j+1}-t_{j}$.

Now, $f_{k}\left(\mathbf{Z}_{k+1}\right)$ at stage $k$ is derived as

$$
\begin{aligned}
f_{k}\left(\mathbf{Z}_{k+1}\right)= & \int_{0}^{\infty} \int_{0}^{\infty} \pi(\psi) f\left(\mathbf{Z}_{k+1} \mid \psi\right) d \psi_{\text {ON }} d \psi_{\text {OFF }} \\
= & \int_{0}^{1} \int_{0}^{\infty} \pi\left(u, \psi_{\text {OFF }}\right) \cdot(1-u)^{1-Z_{t_{1}}} u^{Z_{t_{1}}} \\
& \cdot\left\{\prod_{j=1}^{k} f\left(Z_{t_{j+1}} \mid Z_{t_{j}}, \psi\right)\right\} \cdot\left(\frac{\psi_{\text {OFF }}}{u^{2}}\right) d \psi_{\text {OFF }} d u
\end{aligned}
$$

which provides a closed-form solution by transforming the product of sums with $k$ terms, $\prod_{j=1}^{k} f\left(Z_{t_{j+1}} \mid Z_{t_{j}}, \psi\right)$, into a sum of products with $2^{k}$ terms. We then obtain two estimates $\hat{\psi}_{O N}$ and $\hat{\psi}_{O F F}$ at stage $k$ as in (15) where both of which provide closed-form estimators with the same transformation as in $f_{k}\left(\mathbf{Z}_{k+1}\right)$. The derived Bayesian estimators work as fast as ML estimators since both are expressed in closed forms

$$
\begin{aligned}
\hat{\psi}_{O N, k}= & \int_{0}^{\infty} \int_{0}^{\infty} \psi_{O N} \cdot \pi\left(\psi \mid \mathbf{Z}_{k+1}\right) d \psi_{O N} d \psi_{O F F} \\
= & \int_{0}^{1} \int_{0}^{\infty}\left(\frac{1}{u}-1\right) \frac{\pi\left(u, \psi_{O F F}\right) f\left(\mathbf{Z}_{k+1} \mid \psi\right)}{f_{k}\left(\mathbf{Z}_{k+1}\right)} \\
& \cdot\left(\frac{\psi_{O F F}}{u}\right)^{2} d \psi_{O F F} d u \\
\hat{\psi}_{O F F, k}= & \int_{0}^{\infty} \int_{0}^{\infty} \psi_{O F F} \cdot \pi\left(\psi \mid \mathbf{Z}_{k+1}\right) d \psi_{O N} d \psi_{O F F} \\
= & \int_{0}^{1} \int_{0}^{\infty} \frac{\pi\left(u, \psi_{O F F}\right) f\left(\mathbf{Z}_{k+1} \mid \psi\right)}{f_{k}\left(\mathbf{Z}_{k+1}\right)} \cdot\left(\frac{\psi_{O F F}}{u}\right)^{2} d \psi_{O F F} d u .
\end{aligned}
$$

\subsection{Complexity Reduction}

Despite its good performance, Bayesian inference suffers from computational complexity inherent in integration of pdfs to produce $f_{k}\left(\mathbf{Z}_{k+1}\right)$ and $\hat{\psi}$. In case there are no closedform solutions for them, the complexity grows exponentially as the number of stages increases, although the number of samples increases linearly.

To overcome this problem, we make a few adjustments to the proposed scheme. First, MAX_BS_STAGE, a design parameter, is introduced such that the process resets to stage 1, whenever the current stage number reaches MAX_BS_STAGE. When it resets, the prior distribution $\pi(\psi)$ is updated with the most recent estimates. That is, $\tau_{O N}$ and $\tau_{O F F}$ in $\pi(\psi)$ are replaced by $1 / \hat{\psi}_{O N}$ and $1 / \hat{\psi}_{O F F}$, respectively, where $\hat{\psi}_{O N}$ and $\hat{\psi}_{O F F}$ are the most recent estimates.

Next, a precomputed look-up table is used to evaluate the integrals. When an integration does not provide an analytical
TABLE 2

Test-Specific Simulation Parameters, $i \in\{1, \ldots, M\}$

\begin{tabular}{|l|l|}
\hline Test 1 & Test 2 \\
\hline$M=12$ & $M=18$ \\
\hline$C_{i}=1.0$ for $i \in\{1,2,3\}$ & $C_{i}=1.0$ for $i \in\{1,2,3\}$ \\
$C_{i}=2.5$ for $i \in\{4,5,6\}$ & $C_{i}=2.5$ for $i \in\{4,5,6,7\}$ \\
$C_{i}=4.0$ for $i \in\{7,8,9\}$ & $C_{i}=4.0$ for $i \in\{8,9,10,11\}$ \\
$C_{i}=5.5$ for $i \in\{10,11,12\}$ & $C_{i}=5.5$ for $i \in\{12,13,14,15\}$ \\
& $C_{i}=7.0$ for $i \in\{16,17,18\}$ \\
\hline Test $1(\mathrm{a}): B_{\text {req }}=8, T_{I}^{\max }=25, \bar{u}=0.4-0.6$ \\
Test $1(\mathrm{~b}): B_{\text {req }}=6-10, T_{I}^{\max }=25, \bar{u}=0.4$ \\
Test $1(\mathrm{c}): B_{\text {req }}=8, T_{I}^{\max }=14.5-25, \bar{u}=0.4$ \\
\hline
\end{tabular}

solution, numerical integration (e.g., Simpson's rule) or Monte Carlo integration [7] can be used. Through a series of computations, the estimates of unknown parameters can be precomputed for each possible pair of sample values and their timestamps. This way, the computational complexity of Bayesian estimation can be bounded reasonably small.

\section{Performance Evaluation}

To demonstrate the efficacy of the proposed schemes, we conducted two types of simulation. First, Section 8.1 compares the average opportunity-discovery delay of the proposed near-optimal sequence with the optimal DP-based sequence and previously proposed probabilistic sequences (i.e., sorting channels in descending order of $\theta_{i}$ ) in [2], [9]. Second, Section 8.2 demonstrates the performance improvement of the BCL update strategy compared to the case without BCL update.

The simulation parameters for the tests are summarized in Table 2. For channel $i$, we set

$$
\begin{aligned}
T_{I}^{i} & =T_{I}^{\max }-\left(2 \cdot T_{I}^{\max }-26\right) \cdot(i-1) /(M-1) \text { in msec, } \\
u^{i} & =0.4 \cdot(i-1) /(M-1)+(\bar{u}-0.2),
\end{aligned}
$$

where $T_{I}^{\max }$ and $\bar{u}$ are shown in Table 2 .

Channels are simulated as alternating renewal processes with exponentially distributed $\mathrm{ON}$ and OFF periods. The channel parameters $\psi_{O N}$ and $\psi_{O F F}$ are assumed time varying and increasing/decreasing by 10 percent every 100 seconds. In addition, Test 1 sets $1.0 \leq E\left[T_{O N}^{i}\right] \leq 1.45$ (in seconds) and Test 2 uses 12 channels with $1.0 \leq E\left[T_{O N}^{i}\right] \leq$ 1.45 and six channels with $E\left[T_{O N}^{i}\right] \in\{15,20\}$.

For every test, a single simulation ran for 1,000 seconds, and the same test was repeated 10 times to take the average performance. Other test parameters are set as follows: tRETRY $=0.1, t U P D A T E=5$, thPOTENTIAL $=0.9$, thPOTENTIAL $L_{\text {lower }}=0.88$, thPOTENTIAL $L^{\text {upper }}=0.93$, $N_{\text {lower }}=5$, and $N^{\text {upper }}=10$.

For all tests, we use the average delay as a yardstick in discovering opportunities. The average discovery delay is captured by considering two different cases: 1) when opportunity discovery completes during the first round of searching backup channels (called Type-I delay); and 2) when it completes during the successive retries, provided the first round failed (called Type-II delay). The Type-I delay says how efficient a sensing sequence is, whereas the Type-II delay shows how efficiently the BCL is 


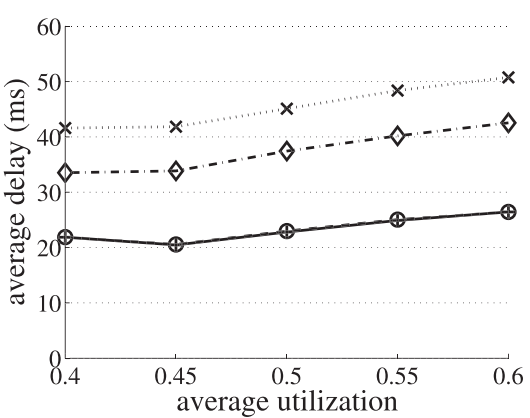

(a) Varying $u^{i}$

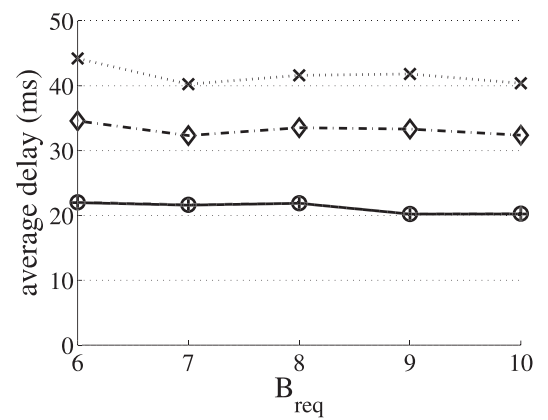

(b) Varying $B_{r e q}$

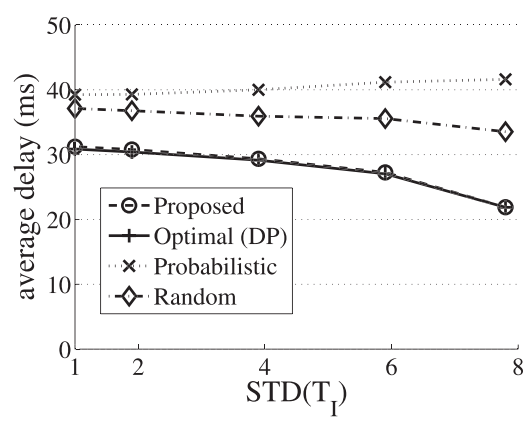

(c) Varying $T_{I}^{i}$

Fig. 8. Test 1: performance of the proposed sequences.

constructed/updated so that opportunity discovery may be successful at early rounds.

In our simulations, we assume perfect estimates and perfect sensing (i.e., $P M D \approx 0$ and $P F A \approx 0$ ) to focus on the efficacy of the proposed sensing sequence and BCL update strategies. It should be noted, however, that this assumption is made only for an illustrative purpose, and our schemes can adopt the schemes in Section 7 for estimation and Section 4.2.1 for imperfect sensing.

\subsection{Test 1: Performance of Proposed Sequence}

In this test, the proposed suboptimal sensing sequence is compared with the optimal sequence given by the DP algorithm, along with the probabilistic sequence that sorts channels in descending order of $\theta_{i}$ and a random sequence. We tested three scenarios where either $\bar{u}, B_{\text {req }}$, or $T_{I}^{\max }$ varies. The case of varying $T_{I}^{\max }$ corresponds to the standard deviation of $T_{I}^{i}$ s varying from 1 to 8 . In addition, no BCL update is performed to focus on the performance of the sequences.

Fig. 8 plots the simulation results. The proposed suboptimal sequence shows a near-optimal performance in all three scenarios incurring only a $0.002-1.25$ percent (average: 0.44 percent) longer delay than the optimal performance. Moreover, the proposed scheme is shown to enhance the delay by 20.4-51.6 percent (average: 42.5 percent) against the probabilistic sequence and by 15.839.3 percent (average: 31.8 percent) against the random sequence. Interestingly, the previously proposed probabilistic sequence performs even worse than the random one in the tested scenario, since the channels with smaller $u^{i}$ (i.e., lower-indexed channels) incur larger sensing-time while

TABLE 3

Impact of Channel-State Conversion in Test 1

\begin{tabular}{|c|c|c|c|c|c|c|}
\hline \multirow{3}{*}{ Test1(a) } & $p_{\text {conv }}$ & 0.045 & 0.055 & 0.082 & 0.10 & 0.13 \\
& $f_{\text {conv }}$ & 1.031 & 1.031 & 1.032 & 1.037 & 1.047 \\
& $f_{\text {model }}$ & 1.047 & 1.058 & 1.089 & 1.115 & 1.151 \\
\hline \multirow{3}{*}{ Test1(b) } & $p_{\text {conv }}$ & 0.025 & 0.035 & 0.045 & 0.052 & 0.055 \\
& $f_{\text {conv }}$ & 1.026 & 1.027 & 1.031 & 1.025 & 1.028 \\
& $f_{\text {model }}$ & 1.026 & 1.036 & 1.047 & 1.055 & 1.059 \\
\hline \multirow{3}{*}{ Test1(c) } & $p_{\text {conv }}$ & 0.045 & 0.050 & 0.055 & 0.055 & 0.053 \\
& $f_{\text {conv }}$ & 1.031 & 1.028 & 1.031 & 1.026 & 1.022 \\
& $f_{\text {model }}$ & 1.047 & 1.052 & 1.058 & 1.059 & 1.056 \\
\hline
\end{tabular}

providing smaller capacities. This shows that the optimal sequence can only be derived by considering all three channel characteristics defined by the tuple of $\left(C_{i}, T_{I}^{i}, \theta_{i}\right)$.

We have also evaluated the probability of channel-state conversion occurrence $\left(p_{\text {conv }}\right)$ and the inflation factor $f_{\text {con }}$, and presented them in Table 3 (each column represents varying $x$-axis values in Fig. 8 ). As shown, state conversion occurs with probability 0.025-0.13 (average: 0.059) and inflates the opportunity-discovery delay only up to 4.7 percent. The impact of state conversion is moderate because sequential sensing is triggered by the vacation of a single in-band channel, and thus, it suffices to discover a few more idle channels-in the test scenarios each sequential sensing senses an average of 4.4 channels. Table 3 also shows the accuracy of the model in (11) by deriving $f_{\text {model }}$ from the measured $p_{\text {conv }}$ and by comparing it with $f_{\text {conv }}$. It is observed that the model overestimates the inflation factor by an average of 3.2 percent.

\subsection{Test 2: BCL Update versus No BCL Update}

In this test, the efficiency of the proposed BCL update strategy is evaluated and compared with another scheme with no BCL update. Both schemes initialize their BCL assuming no prior knowledge on the ON/OFF usage patterns. As the simulation progresses, however, the proposed scheme updates BCL via channel import/ export/swap and adjusts the BCL size accordingly, whereas the latter scheme always stays with its initial BCL entries. In addition, both schemes use the proposed suboptimal sequence.

In Fig. 9, we plot the average "overall" delay (i.e., Type-I and Type-II combined) with the delay-type ratio between
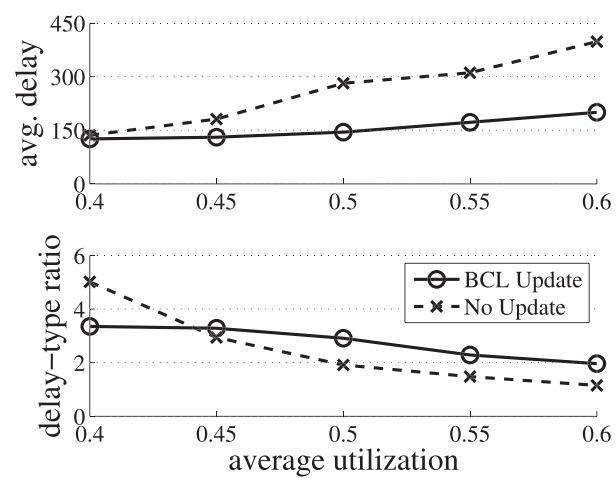

Fig. 9. Test 2: Proposed BCL update versus no update. 
the number of Type-I events and that of Type-II events. The proposed BCL update scheme flexibly adjusts its BCL size from 5 to 10 to maximize the chance of successful opportunity discovery at the first round of sequential sensing, and thus, the BCL update case incurs more Type-I events than Type-II events compared to the no-update case (as shown for $\bar{u} \geq 0.45$ ) reducing the overall delay by 7.3-49.6 percent (average: 35.6 percent). This is because the Type-II delay is more costly due to the huge delays in retrying sequential sensing.

\section{Conclusions}

In this paper, we proposed a DP-based optimal sensingsequence and a computationally efficient near-optimal sensing-sequence for fast discovery of spectrum opportunities to promote seamless service provisioning of CRNs while minimizing QoS degradation. To support the proposed fast opportunity discovery mechanism, we also proposed an efficient BCL management algorithm and a hybrid estimation strategy of ML and Bayesian inference for reliable estimation of channel-usage patterns.

In future, we plan to extend the problem to the scenario, where colocated CRNs have overlapping channels. In such a case, a CRN should treat its local-only channels and shared channels differently in sorting channel, to avoid collision between CRNs.

\section{ACKNOWLEDGMENTS}

The work reported in this paper was supported in part by the US National Science Foundation under Grants CNS 0519498 and 0721529, by Intel Corporation, and by the 1.110033.01 Research Fund of the Ulsan National Institute of Science and Technology (UNIST). An earlier version of this work was presented at IEEE DySPAN 2008 [1].

\section{REFERENCES}

[1] H. Kim and K.G. Shin, "Fast Discovery of Spectrum Opportunities in Cognitive Radio Networks," Proc. IEEE Third Symp. New Frontiers in Dynamic Spectrum Access Networks (DySPAN), Oct. 2008

[2] H. Kim and K.G. Shin, "Efficient Discovery of Spectrum Opportunities with MAC-Layer Sensing in Cognitive Radio Networks," IEEE Trans. Mobile Computing, vol. 7, no. 5, pp. 533545, May 2008.

[3] T. Shu and M. Krunz, "Throughput-Efficient Sequential Channel Sensing and Probing in Cognitive Radio Networks Under Sensing Errors," Proc. ACM MobiCom, Sept. 2009.

[4] L. Lai, H.E. Gamal, H. Jiang, and H.V. Poor, "Cognitive Medium Access: Exploration, Exploitation and Competition," IEEE Trans. Mobile Computing, vol. 10, no. 2, pp. 239-253, Feb. 2011.

[5] H. Kim and K.G. Shin, "In-Band Spectrum Sensing in Cognitive Radio Networks: Energy Detection or Feature Detection?" Proc. ACM MobiCom, Sept. 2008.

[6] IEEE 802.22 Working Group on Wireless Regional Area Networks, http:/ / www.ieee802.org/22, 2013.

[7] J.O. Berger, Statistical Decision Theory and Bayesian Analysis, second ed. Springer Science, 2006.

[8] N.B. Chang and M. Liu, "Optimal Channel Probing and Transmission Scheduling for Opportunistic Spectrum Access," Proc. ACM MobiCom, Sept. 2007.

[9] H. Jiang, L. Lai, R. Fan, and H.V. Poor, "Optimal Selection of Channel Sensing Order in Cognitive Radio," IEEE Trans. Wireless Comm., vol. 8, no. 1, pp. 297-307, Jan. 2009.
[10] S.H.A. Ahmad, M. Liu, T. Javidi, Q. Zhao, and B. Krishnamachari, "Optimality of Myopic Sensing in Multichannel Opportunistic Access," IEEE Trans. Information Theory, vol. 55, no. 9, pp. 40404050, Sept. 2009.

[11] R. Fan and H. Jiang, "Channel Sensing-Order Setting in Cognitive Radio Networks: A Two-User Case," IEEE Trans. Vehicular Technology, vol. 58, no. 9, pp. 4997-5008, Nov. 2009.

[12] H.T. Cheng and W. Zhuang, "Simple Channel Sensing Order in Cognitive Radio Networks," IEEE J. Selected Areas Comm., vol. 29, no. 4, pp. 676-688, Apr. 2011.

[13] A. Motamedi and A. Bahai, "MAC Protocol Design for SpectrumAgile Wireless Networks: Stochastic Control Approach," Proc. IEEE Second Int'l Symp. New Frontiers in Dynamic Spectrum Access Networks (DySPAN), Apr. 2007.

[14] J. Wang, M.S. Song, S. Santhiveeran, K. Lim, G. Ko, K. Kim, S.H. Hwang, M. Ghosh, V. Gaddam, and K. Challapali, "First Cognitive Radio Networking Standard for Personal/Portable Devices in TV White Spaces," Proc. IEEE Int'l Symp. New Frontiers in Dynamic Spectrum Access Networks (DySPAN), Apr. 2010.

[15] N. Nie and C. Comaniciu, "Adaptive Channel Allocation Spectrum Etiquette for Cognitive Radio Networks," Mobile Networks and Applications, vol. 11, no. 6, pp. 779-797, Dec. 2006.

[16] C.-T. Chou, N. Sai Shankar, H. Kim, and K.G. Shin, "What and How Much to Gain by Spectral Agility," IEEE J. Selected Areas Comm., vol. 25, no. 3, pp. 576-588, Apr. 2007.

[17] R. Rajbanshi, Q. Chen, A.M. Wyglinski, G.J. Minden, and J.B. Evans, "Quantitative Comparison of Agile Modulation Techniques for Cognitive Radio Transceivers," Proc. IEEE Fourth Consumer Comm. Networking Conf. (CCNC), Jan. 2007.

[18] H. Kim, C. Cordeiro, K. Challapali, and K.G. Shin, "An Experimental Approach to Spectrum Sensing in Cognitive Radio Networks with Off-the-Shelf IEEE 802.11 Devices," Proc. IEEE Fourth Consumer Comm. Networking Conf. (CCNC), Jan. 2007.

[19] C. Cordeiro and K. Challapali, "C-MAC: A Cognitive MAC Protocol for Multi-Channel Wireless Network," Proc. IEEE Second Int'l Symp. New Frontiers in Dynamic Spectrum Access Networks (DySPAN), Apr. 2007.

[20] B. Hamdaoui and K.G. Shin, "OS-MAC: An Efficient MAC Protocol for Spectrum-Agile Wireless Networks," IEEE Trans. Mobile Computing, vol. 7, no. 8, pp. 915-930, Aug. 2008.

[21] S. Geirhofer, L. Tong, and B.M. Sadler, "Dynamic Spectrum Access in the Time Domain: Modeling and Exploiting White Space," IEEE Comm. Mag., vol. 45, no. 5, pp. 66-72, May 2007.

[22] D.R. Cox, Renewal Theory. Butler \& Tanner, 1967.

[23] C. Cordeiro, K. Challapali, and M. Ghosh, "Cognitive PHY and MAC Layers for Dynamic Spectrum Access and Sharing of TV Bands," Proc. ACM First Int'l Workshop Technology and Policy for Accessing Spectrum (TAPAS), Aug. 2006.

[24] A. Ghasemi and E.S. Sousa, "Collaborative Spectrum Sensing for Opportunistic Access in Fading Environments," Proc. IEEE First Int'l Symp. New Frontiers in Dynamic Spectrum Access Networks (DySPAN), Nov. 2005.

[25] G. Ganesan and Y. Li, "Cooperative Spectrum Sensing in Cognitive Radio Networks," Proc. IEEE First Int'l Symp. New Frontiers in Dynamic Spectrum Access Networks (DySPAN), Nov. 2005.

[26] S.M. Mishra, A. Sahai, and R.W. Brodersen, "Cooperative Sensing among Cognitive Radios," Proc. IEEE Int'l Conf. Comm. (ICC), June 2006.

[27] D. Simon, Optimal State Estimation: Kalman, $\mathrm{H}_{\infty}$, and Nonlinear Approaches. John Wiley \& Sons, 2006.

[28] C. Cordeiro, K. Challapali, D. Birru, and N. Sai Shankar, "IEEE 802.22: An Introduction to the First Wireless Standard Based on Cognitive Radios," J. Comm., vol. 1, no. 1, pp. 38-47, Apr. 2006. 


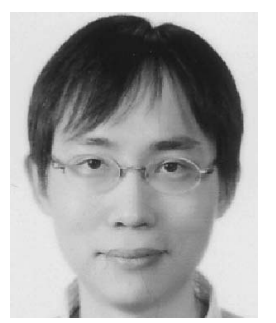

Hyoil Kim received the BS degree in electrical engineering from Seoul National University, Korea, in 1999, and the MS and PhD degrees in electrical engineering systems from the University of Michigan in 2005 and 2010 , respectively. Since August 2011, he has been an assistant professor at the School of Electrical and Computer Engineering, Ulsan National Institute of Science and Technology (UNIST), Korea. Before joining UNIST, he was a postdoctoral researcher in the IBM T.J. Watson Research Center, Hawthorne, New York, in 2010-2011. His research interests include cognitive radios, dynamic spectrum access, mobile cloud computing, and MANETs. He served as a program committee member of the IEEE GlobeCom Wireless Networking Symposium (2011-2013), ICUFN 20122013, ACM WiNTECH 2013, and IEEE INFOCOM 2014. He is a member of the IEEE.

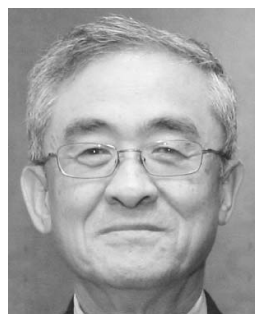

Kang G. Shin is the Kevin \& Nancy O'Connor professor of computer science in the Department of Electrical Engineering and Computer Science, The University of Michigan, Ann Arbor. His current research focuses on computing systems and networks as well as on embedded real-time and cyber-physical systems, all with an emphasis on timeliness, security, and dependability. He has supervised the completion of $71 \mathrm{PhDs}$ and authored/coauthored more than 770 technical articles (more than 270 of these are in archival journals), one textbook, and more than 20 patents or invention disclosures. He has received numerous awards, including best paper awards from the 2011 ACM Annual International Conference on Mobile Computing and Networking (MobiCom '11), the 2011 IEEE International Conference on Autonomic Computing, and the 2010 and 2000 USENIX Annual Technical Conferences, as well as the 2003 IEEE Communications Society William R. Bennett Prize Paper Award and the 1987 Outstanding IEEE Transactions of Automatic Control Paper Award. He has also received several institutional awards, including the Research Excellence Award in 1989, the Outstanding Achievement Award in 1999, the Distinguished Faculty Achievement Award in 2001, and the Stephen Attwood Award in 2004 from The University of Michigan (the highest honor bestowed to Michigan Engineering faculty); a Distinguished Alumni Award of the College of Engineering, Seoul National University in 2002; the 2003 IEEE RTC Technical Achievement Award; and the 2006 Ho-Am Prize in Engineering (the highest honor bestowed to Korean-origin engineers). He has chaired several major conferences, including 2009 ACM MobiCom, 2008 IEEE SECON, 2005 ACM/USENIX MobiSys, 2000 IEEE RTAS, and 1987 IEEE RTSS. He served on editorial boards including the IEEE Transactions on Parallel and Distributed Systems and ACM Transactions on Embedded Systems. He has also served or is serving on numerous government committees, such as the US National Science Foundation CyberPhysical Systems Executive Committee and the Korean Government R\&D Strategy Advisory Committee. He has also cofounded a couple of startups. He is a fellow of the IEEE and ACM.

$\triangleright$ For more information on this or any other computing topic, please visit our Digital Library at www.computer.org/publications/dlib. 